\title{
Implications of multi-asperity contact for shear stress distribution in the viable epidermis - An image-based finite element study.
}

Maria F. Leyva-Mendivil ${ }^{1,2}$, Jakub Lengiewicz ${ }^{3}$, Anton Page ${ }^{4}$, Neil W. Bressloff ${ }^{5}$ and Georges Limbert ${ }^{1,2,6^{*}}$

${ }^{1}$ national Centre for Advanced Tribology at Southampton (nCATS), Faculty of Engineering and the Environment, University of Southampton. Southampton, SO17 1BJ, UK.

${ }^{2}$ Bioengineering Science Research Group, Faculty of Engineering and the Environment, University of Southampton. Southampton, SO17 1BJ, UK.

${ }^{3}$ Institute of Fundamental Technological Research, Polish Academy of Sciences (IPPT PAN), ul. Pawinskiego 5B; 02-106 Warsaw, Poland

${ }^{4}$ Biomedical Imaging Unit, Faculty of Medicine, University of Southampton, Southampton General Hospital, Southampton, SO16 6YDJ, UK.

${ }^{5}$ Computational Engineering and Design Group, Faculty of Engineering and the Environment, University of Southampton. Southampton, SO17 1BJ, UK.

${ }^{6}$ Laboratory of Biomechanics and Mechanobiology, Division of Biomedical Engineering, Department of Human Biology, Faculty of Health Sciences, University of Cape Town, Observatory 7935, South Africa.

\section{Abstract}

Understanding load transfer mechanisms from the surface of the skin to its deeper layers is crucial in gaining a fundamental insight into damage phenomena related to skin tears, blisters and superficial/deep tissue ulcers. It is unknown how shear stresses in the viable epidermis are conditioned by the skin surface topography and internal microstructure and to which extent their propagation is conditioned by the size of a contacting asperities.

In this computational study, these questions were addressed by conducting a series of contact finite element analyses simulating normal indentation of an anatomically-based two-dimensional multi-layer model of the skin by rigid indenters of various sizes and sliding of these indenters over the skin surface. Indentation depths, local (i.e. microscopic) coefficients of friction and Young's modulus of the stratum corneum were also varied. For comparison purpose and for isolating effects arising purely from the skin microstructure, a geometrically-idealised equivalent multi-layer model of the skin was also considered.

The multi-asperity contact induced by the skin topographic features in combination with a non-idealised geometry of the skin layers lead to levels of shear stresses much higher than those produced in the geometrically-idealised case. These effects are also modulated by other system parameters (e.g. local coefficient of friction, indenter radius).

These findings have major implications for the design and analyses of finite element studies aiming at modelling the tribology of skin, particularly if the focus is on how surface shear stress leads to damage initiation which is a process known to occur across several length scales.

\section{Key words}

Skin, microstructure, contact mechanics, indentation, sliding contact, finite element, image-based modelling, material properties

\begin{tabular}{|ll}
\hline Journal: & Biotribology \\
Article type: & Research Article \\
Date: & Revision 1, 2 March 2017 \\
*Corresponding author: & Georges Limbert \\
& E-mail address: g.limbert@soton.ac.uk \\
\hline
\end{tabular}




\section{Introduction}

The multi-faceted protective role of human skin is undeniable: as a sophisticated living biophysical interface, the skin provides thermal, chemical, biological, radiological and mechanical protection to the body [1]. These functional abilities are strongly linked to the skin multiscale structural hierarchy. At the mesostructural level the skin can be viewed as a three-layer structure formed of adipose tissue (hypodermis), fibrous tissue (dermis), and epithelial cells (epidermis) from the inside out. In the upper dermis (papillary dermis), capillary blood vessels carry nutrients to the epidermis. Epithelial cells in the basal layer separating the viable epidermis from the papillary dermis replicate and push older cells towards the surface in a constant renewal process which spans 10 to 30 days. As these cells are driven away from the source of nutrients, they undergo a keratinisation process to eventually form a 15-20 cell-thick layer of (dead) flat corneocytes know as stratum corneum [2]. These corneocytes are strongly bonded by cellular joints called desmosomes, which maintain the integrity of this layer in most contact interactions. With degradation of the desmosomes of the oldest corneocytes, desquamation takes place. The stratum corneum provides the interface for the external skin contact interactions and the physico-chemical properties of this layer have a direct effect on skin friction [3]. The structure of the stratum corneum allows penetration of water, lipids and other substances (e.g. body lotion, ointments) into the intercellular space. Increasing relative humidity does not only lead to swelling of the stratum corneum [4] but also modifies its mechanical properties and makes it more sensitive to temperature fluctuations. As a consequence, both relative humidity and temperature (mutually represented by the notion of micro-climate) can also affect how strain and stress are propagated to the skin layers laying beneath the stratum corneum, the nature and intensity of contact interactions (i.e. friction) [5-7] and the likelihood of developing skin injuries [8, 9].

The skin contains sensory receptors that detect deformations, vibrations and changes in temperature. This encoded information is transmitted through neuronal pathways to the brain where the information is processed. In contact interactions, mechano-receptors provide information about the characteristics of the surface contacting the skin and the magnitude of deformation. As illustrated in Fig. 1, mechano-receptors are distributed throughout the dermis while nociceptors throughout the epidermis $[1,10]$. If the skin experiences excessiveand therefore potentially hazardous-deformations, nociceptors release an electro-chemical "pain" signal, urging the brain to take action to remove that source of pain and, as a consequence prevent any initiation or extension of damage (withdrawal reflex) [11]. However, if the neuronal pathway is disrupted, the pain signal does not reach the brain, increasing the risk of skin damage. This situation occurs in the case of comatose patients and those with paralysis or peripheral neuronal damage (e.g. diabetic patients) whose sensory perception of contact-related skin deformations is lacking or significantly decreased. In these situations, any prolonged position which involves contact pressure and shear at the surface of the skin has the potential to be seriously detrimental to patient's skin integrity which can ultimately result in the occurrence of superficial and deep pressure sores [12-14].

In the last decade, unravelling and studying the mechanisms behind skin injuries has become a topic of great scientific and societal interest. It is known that repetitive loading of skin, especially under shear, results in skin lesions such as friction blisters [15]. Also, prolonged application of pressure can result in skin and deep tissue damage (pressure ulcers), which is aggravated when combined with interfacial shear [16-18]. In these situations, the interfacial properties of the skin and counter surface (e.g. adhesion, topography of micro-asperities) determine the level of traction skin experiences, and such traction has proven to be related to the likelihood of developing a skin injury [5]. However, friction-related skin damage often starts beneath the stratum corneum: friction blisters are formed by fatigue failure of the inter-cellular joints (desmosomes) in the viable epidermis [19]; and stage I pressure ulcers (skin damage with no visible break down) involve the obstruction and reperfusion of the capillary blood flow in the papillary dermis (under the viable epidermis) [20].

A recent study by $\mathrm{Wu}$, van der Schaft, Baaijens and Oomens [21] showed that the application of shear and compressive stresses to muscle cells can induce cell death. Pig model studies have also shown that interfacial shear up to $70 \mathrm{kPa}$ can also result in skin breakdown [22]. However, a question that has not yet been answered is how shear stresses are transmitted from the skin surface to the skin epithelial cells. Could shear stress in the viable epidermis contribute to stage I pressure ulcers? An attempt to answer this question was set by Sopher and Gefen [9] by means of a finite element model representing the stratum corneum as an undulated surface. In their study, they evaluated sensitivity of shear stress distribution to three parameters: coefficient of friction of skin against a surface, shear modulus and thickness of the stratum corneum, herein considered to be mechanically homogeneous. 
However, their geometrically idealised representation of the skin did not capture the effect of the skin topography and microstructure on the distribution of internal shear stress. In a previous study [23], we developed an image-based two-dimensional (2D) finite element model of the skin to investigate the role of the skin microstructure in modulating macroscopic strains at the local (i.e. microscopic) level. The computational analyses confirmed the significant effects of skin microstructure in reducing or amplifying macroscopic strains. It was also revealed how the stratum corneum, despite its very small thickness compared to that of the other layers, can play a major role in conditioning the distribution and magnitude of strains in the viable epidermis and dermis.

In the present study, we used the anatomical skin model of Leyva-Mendivil et al. [23] in the context of a recently developed contact modelling framework [24] with the objective of evaluating the role of the skin topography and microstructure on the distribution of shear stress within the viable epidermis. This latter layer contains nociceptors and is also the prime location where cellular damage processes are likely to occur in response to mechanical loads.

The analysis was performed for normal and sliding indentation contact simulations of a rigid indenter over the skin surface. The study considered three different scales of interaction with variation of indenter size, and different physico-chemical conditions at the contact interface implicitly modelled by variation of the coefficient of friction as well as variations in the Young's modulus of the stratum corneum. One of the key novel aspects of this study is the inclusion of multi-asperity contact provided by anatomical skin topographic features-a feature that has been widely overlooked in skin tribology research where the skin is typically assumed to have a smooth surface or a surface roughness that is deemed not to play a role in asperity deformation-induced friction $[3,8$, 25].

\section{Materials and methods}

For this computational study, finite element techniques were used to simulate 2D skin contact interactions with a rigid discoidal indenter of various sizes, effectively capturing different spatial scale of interactions. A plane strain assumption, which is valid for structures which are infinitely long in one direction [26], was used. The focus of the study was to investigate how the skin surface and internal structural topography condition the transmission and propagation of shear stress induced by a rigid slider. The plane strain assumption combined with a 2D formulation was a good compromise between simplicity of geometry and sufficient sensitivity to demonstrate material-structural effects on the shear stress response of skin. In our study, it was assumed that the skin section is infinitely long along the direction normal to the histological section. The 2D discoidal indenter was therefore the equivalent of a single infinitely long cylindrical asperity laying on a flat surface featuring a certain degree of roughness (i.e. skin asperities).

Two types of analyses were considered: normal indentation and sliding indentation. By simulating normal indentation, we quantified the effect of multi-asperity contact on the shear stress distribution in the viable epidermis and compared it to that computed in a geometrically-idealised, multi-layer flat skin model. For both types of model frictionless contact conditions were enforced. For sliding contact, the shear stress distribution in the viable epidermis was used for the calculation of two shear stress metrics: intensity and scattering (to be defined in Section 2.4). These metrics were used to analyse the differences between the shear stress distribution and magnitude observed in the geometrically-idealised skin model and those observed in the anatomical skin model during sliding over the skin anatomical topographic features. The latter analysis was devised to investigate how shear stress distribution within the viable epidermis was affected by: $a$ ) indenter radius and indentation depth, b) local coefficient of friction, and c) stratum corneum Young's modulus (assumed to be implicitly correlated with relative humidity conditions).

\subsection{Multi-layer finite element models of the skin}

The 2D anatomical skin model was constructed from histological images of human skin (a mid-back skin sample obtained from a healthy 30 years old Caucasian female). The details of the procedure followed for sample preparation, image acquisition, image segmentation and finite element meshing can be found in Leyva-Mendivil, Page, Bressloff and Limbert [23]. The finite element model captured the intricate geometry of the stratum corneum, viable epidermis and dermis and resulted in a $1.9281 \mathrm{~mm}$ long sample. In order to avoid the occurrence of boundary effects likely to affect the mechanical response of the skin, the generic model of Leyva-Mendivil, Page, Bressloff and Limbert [23] was extended at its lateral and bottom boundaries using the mean height of each layer resulting in a new model covering a $7 \mathrm{~mm} \times 5 \mathrm{~mm}$ area (see Fig. 2). 
These dimensions were selected according to the recommendations of Karduna, Halperin and Yin [27] for a maximum skin displacement of $0.25 \mathrm{~mm}$ at the top centre of the skin model. The section containing the anatomical features of skin was designated as the region of interest, and only the results in this area of the model were analysed.

The general-purpose finite element application suite Abaqus 6.14 (Simulia, Dassault Systèmes, Providence, RI, USA) was used to generate the mesh of the finite element models. The idealised model contained 151,127 linear triangular elements, with a characteristic element length varying from $2 \mu \mathrm{m}$ in the stratum corneum to $150 \mu \mathrm{m}$ at the base of the region of interest. The anatomical model required further refinement to faithfully capture skin deformation, so that the final element size in the stratum corneum was set to $1.5 \mu \mathrm{m}$ leading to a total of 336,224 plane-strain elements.

The finite element meshes were exported to the symbolic/numeric finite element environment package AceGen/AceFEM [28, 29] integrated within Mathematica ${ }^{\circledR}$ (Wolfram Research, Inc., Champaign, IL, USA.) for the computational analysis of the skin contact interactions. The skin layers were modelled as neo-Hookean hyperelastic solids defined by a strain energy function $\psi$, as in previous studies [23]:

$$
\psi=\frac{E}{4(1+v)}\left[J^{-2 / 3}(\mathbf{C}: \mathbf{I})-3\right]+\frac{E}{6(1-2 v)}(J-1)^{2}
$$

where $\mathbf{C}$ is the right Cauchy-Green deformation tensor, $\mathbf{I}$ the second-order identity tensor and $J=\sqrt{\operatorname{det}(\mathbf{C})}$, the third invariant of $\mathbf{C}$ [30]. $E$ and $v$ are respectively the ground state (or initial) Young's modulus and Poisson's ratio of the material.

\subsection{General parametric contact modelling approach}

To characterise the multi-factorial shear stress response of the skin viable epidermis a parametric study was conducted by considering the following variables:

- Type of contact interaction: normal indentation or sliding indentation

- Indenter radius $R_{1}: 0.1 \mathrm{~mm}, 0.25 \mathrm{~mm}, 0.5 \mathrm{~mm}$;

- Local coefficient of friction $\mu_{1}$ between skin and indenter: $0.0,0.1,0.2,0.3$;

- Young's modulus of the stratum corneum $E_{S C}: 0.6 \mathrm{MPa}, 370 \mathrm{MPa}$

Indenter sizes were selected according to probe diameters reported in the literature for skin indentation (e.g. [31-33]). The local coefficients of friction were selected to be within the range of experimental values reported in the literature for both dry and wet contact of skin and various materials (e.g. Nylon, PTFE, PVC, polyethylene, polycarbonate, glass, aluminium, steel) as reported in review paper on skin tribology by Derler and Gerhardt [8]. Although these conditions were set according to the macroscopic friction reported in the literature, they do not necessarily capture the same global friction response reported in the literature [24]. The Young's moduli of the stratum corneum were selected to be representative of 100 and $30 \%$ relative humidity conditions [34], and the Poisson's ratio was set to $v_{S C}=0.3$, which is within the range of compressibility accepted for skin tissue [3539].

\subsubsection{Analysis variants}

A total of 60 analyses were performed for the anatomical and idealised skin models to account for different combinations of contact conditions (normal indentation and sliding indentation, indenter radius, coefficient of friction and Young's modulus of the stratum corneum). For the case of normal indentation, only the effects of multi-asperity contact on the shear stress distribution were considered, but not those of the local friction. Taking the Hertzian contact model as a reference, as explained in Section 2.4, these simulations were performed with frictionless local contact conditions (i.e. $\mu_{l}=0.0,12$ analyses). For sliding contact study four different values of local coefficient of friction were considered resulting in 48 analyses.

Rather than a comparison of results for all combination of variables, this study analysed the results by considering the following distinct cases:

1. Normal indentation: sensitivity of the shear stress distribution to model geometry, indenter size and mechanical properties of the stratum corneum.

a) Analytical (Hertzian) model vs homogeneous flat model (validation)

b) Anatomical vs Idealised skin model

2. Sliding indentation: sensitivity of the shear stress distribution to model geometry, contact conditions, indenter size and mechanical properties of the stratum corneum.

a) Idealised skin model

b) Anatomical vs Idealised skin model at the different topographic features 


\subsubsection{Mechanical properties}

The dermis and viable epidermis were assumed to have identical mechanical properties for all of the simulations. Both neo-Hookean layers were assigned the following identical mechanical properties: a $E_{D}=E_{V E}=0.6 \mathrm{MPa}$ Young's modulus and a Poisson's ratio $v_{D}=v_{V E}=0.3=v_{S C}[32,40,41]$.

\subsubsection{Fixed boundary conditions}

The skin models (implemented using a plane-strain formulation) were defined in a 2D $x y$ frame. The $x$-axis is parallel to the base of the skin model and the mean contact surface. The $y$-axis is orthogonal to the $x$-axis (see Fig. 2). The base of the skin models was rigidly fixed while the lateral model edges were unconstrained.

\subsubsection{Displacement conditions for normal indentation and sliding indentation}

The indenter was modelled as a discoidal rigid body. The centre of the indenter was aligned in the $x$-direction with the centre of the region of interest for normal indentation, and at $1 \mathrm{~mm}$ from the centre of the skin model for the sliding contact. In the $y$-direction, the centre of the indenter was aligned 1.1 times its radius from the highest point of the skin surface (see Fig. 2). A vertical displacement $D_{y} \leq R_{1} / 2$ was imposed for normal indentation simulations at the centre of the skin model, to avoid boundary effects [27]. Sliding contact was simulated in two steps by imposing first a vertical displacement of $D_{y}=R_{1} / 2$ from the mean surface height, followed by a lateral displacement $D_{x}=2.0 \mathrm{~mm}$ towards the right vertical edge of the model (sliding over the whole region of interest). A constant local coefficient of friction $\mu_{l}$ was used throughout the two steps of the simulation (indentation followed by sliding).

\subsubsection{Contact formulation}

The finite element contact formulation was based on an augmented Lagrangian method featuring Coulomb friction, with the definition of a local coefficient of friction $\mu_{l}$, following the approach developed by Lengiewicz, Korelc and Stupkiewicz [42]. Due to the complexity of the skin structure coupled to structural instabilities arising from the extremely large ratio of stiffness between the skin and indenter, the convergence of the numerical analysis proved very challenging as snap-through type phenomena were observed, especially during sliding contact. In order to regularise the analyses and ensure convergence of the results, a dissipative Newmark scheme was adopted [43]. The Newmark parameters were adjusted to improve convergence of the solution while ensuring that dynamic effects likely to perturb the quasi-static solution were negligible. This approach was implemented within the AceGEN/AceFEM system using a Newton-Raphson solving technique [29, 42]. It is relevant to point out that a contact algorithm for self-contact of the outer surface of the skin was not used. The inclusion of self-contact to the contact algorithm would significantly increase computational times and, considering the highly non-linear nature of the sliding of a rigid indenter over a very soft surface featuring very high gradient of curvatures, would prove to be a very challenging instable problem to solve.

\subsection{Analytical validation of the idealised finite element models}

The contact analysis procedure for the idealised skin finite element model was validated against an analytical model of Hertzian contact between parallel cylinders, i.e. line contact, corresponding to the 2D plane strain problem presented in this study [44]. The discoidal indenter is defined by a radius $R_{1}$ and elastic properties $\left(E_{1}, v_{1}\right)$. The skin, modelled as a homogeneous half space (idealised skin model), is defined by a radius $R_{2} \rightarrow \infty$ and elastic properties $\left(E_{2}, v_{2}\right)$.

The effective radius of curvature $R^{*}$ is established as:

$$
\frac{1}{R^{*}}=\frac{1}{R_{1}}+\frac{1}{R_{2}}
$$

which, for this study is equivalent to $R^{*}=R_{1}$. The effective Young's modulus for the contact interaction is given by:

$$
\frac{1}{E^{*}}=\frac{1-v_{1}^{2}}{E_{1}}+\frac{1-v_{2}^{2}}{E_{2}}
$$

which, for a rigid indenter (i.e. $E_{1} \rightarrow \infty$ ) simplifies to $E^{*}=E_{2} / 1-v_{2}^{2}$.

In line contact problem, the geometry of the contact area is characterised by the half-width parameter $b$ so the contact area is $2 b \times L$ where $L$ is the length of the contacting cylinders. The analytical validation of the finite element model was performed by comparing the observed half-width in the simulations to the analytical halfwidth, calculated as a function of the normal component of the indenter reaction force $F_{N}$ : 


$$
b=\sqrt{\frac{4 R^{*} F_{N}}{\pi L E^{*}}}
$$

This Hertzian model provides an estimate of the deflection $\delta$ of the material surfaces in line contact between the parallel cylinders as:

$$
\delta=0.319\left(\frac{F_{N}}{L E^{*}}\right) \times\left[\frac{2}{3}+\operatorname{Ln}\left(\frac{4 R_{1} R_{2}}{b^{2}}\right)\right]
$$

However, this equation is not applicable for line contact with a half space (in our problem, the skin) [45], as $\lim \delta_{R_{2} \rightarrow \infty}=\infty$. This problem has been addressed by several authors, as reviewed in the supplementary material of Leyva-Mendivil et al. [24]. For the validation of deflection, we used the analytical model described by Johnson [46] instead, which, using Hertzian contact assumptions, provides an explicit expression of the deflection of the half-space with respect to a point located directly underneath the indenter contact point, at a distance $\zeta$. This length $\zeta$ can be considered to represent the thickness of the skin model. This model predicts deflection as:

$$
\delta=\frac{F_{N}\left(1-v_{2}^{2}\right)}{L \pi E_{2}}\left[2 \ln \left(\frac{2 \zeta}{b}\right)-\frac{v_{2}}{1-v_{2}}\right]
$$

For the validation study, a total of 75 indentation simulations were performed with pseudo-random variations of $E_{2}, R_{1}$ and skin deflection $\delta$, using a low-discrepancy Sobol sequence [47] from the Intel ${ }^{\circledR}$ Math Kernel Library (MKL) generator built-in in Mathematica ${ }^{\circledR}$ (Wolfram Research, Inc., Champaign, IL, USA). The skin effective Young's modulus was varied from 0.01 to $400 \mathrm{MPa}$, the indenter radius from 0.05 to $0.5 \mathrm{~mm}$, and the indentation skin deflection from 0.01 to $0.5 \mathrm{~mm}$.

The two models described above are restricted to frictionless elastic contact interactions between two smooth bodies, where the area of contact is much smaller than the dimensions of the contacting bodies and their effective radius $[44,46]$. This was reflected in the results, where the error between the simulation results and the analytical model increased with higher deformation (see Fig. 5). Nevertheless, for skin deflection lower than $1 \mathrm{~mm}$, the mean relative errors between the finite element simulations and the analytical models were of $4.3 \%$ for $b$ and $5.43 \%$ for $\delta$. These values were sufficiently low to consider the finite element model valid for the more complex simulations with the anatomical model.

\subsection{Post-processing analysis of shear stress distribution}

Shear stress in the viable epidermis was taken as a surrogate measure of likelihood of tissue damage. This output response was measured at each of the elements of the viable epidermis mesh (resulting in a cloud of data points) and plotted with respect to their geometrical location in the $x$ direction of the skin model. These plots (Fig. 6Fig. 9) provide an overview of the range of shear stresses experienced along the skin and throughout the thickness of this layer. The results were analysed with respect to indenter location in the idealised model, and with respect to both indenter location and skin topographical features (i.e. asperities) in the anatomical model. For normal indentation, the results were compared at same indentation depth for different indenter radii. These analyses only compared the effect of indenter radius and stratum corneum's Young's modulus on the shear stress distribution and intensity. For sliding contact, values of shear stress were sampled at different indenter locations corresponding to characteristic topographic features of the skin as shown in Fig. 3 (see also [23]) to highlight their role in conditioning shear stress propagation and distribution in the viable epidermis.

The results section is organised so that normal indentation results are first presented, followed by the sliding indentation ones. In both cases, we present an analysis of the anatomical conditions and compare these results to those corresponding to the idealised ones. From the shear stress distribution data, we established two novel metrics hereby called the intensity and scattering of shear stress that were calculated for each of the simulations. These quantities are defined below.

The shear stress intensity vector was composed of the minimum and maximum shear stress obtained in the viable epidermis as its two scalar components:

$$
\mathbf{a}=\left\{\tau_{\max }, \tau_{\min }\right\}
$$

By defining a shear stress threshold at which skin would be damaged, the intensity vector can be used to represent the likelihood of development of a skin lesion. 
On $10 \%$ of the analysed results, it was observed that large deformations-mostly at topographic feature G (Fig. 3) would cause interpenetration of the skin crests as no self-contact formulation was implemented. This resulted in unrealistic high stress values at the base of the skin furrows. For these cases, the shear stress intensity vector was obtained by considering stress values above and below respectively the 0.2 and 99.8 percentiles.

The shear stress scattering vector $\mathbf{s}$ was composed of the distances at which at least one of the nodes in the viable epidermis reached $10 \%$ of $\tau_{\min }$ behind the indenter $\left(s_{1}\right.$, negative value) and $10 \%$ of $\tau_{\max }$ ahead the indenter $\left(s_{2}\right.$, positive value):

$$
\mathbf{S}=\left\{s_{1}, s_{2}\right\}
$$

This was done to capture the $90^{\text {th }}$ percentile of shear stress distribution without considering extremely low values filling the remaining $10 \%$ of the distribution. The range of shear stress values in the viable epidermis for the idealised model (denoted by the superscript $I M$ ) is given by $\left|a_{1}^{I M}\right|+\left|a_{2}^{I M}\right|$ while the range of shear stress defined by the scattering vector $\mathbf{s}$ is simply $\left|s_{1}^{I M}\right|+\left|s_{2}^{I M}\right|$. To compare the shear stress intensity and scattering vectors for the idealised and anatomical skin models these two vectors for the anatomical model were normalised into $\hat{\mathbf{a}}$ and $\hat{\mathbf{s}}$, the shear stress intensity and scattering vector ratios as follows:

$$
\hat{\mathbf{a}}=\frac{\mathbf{a}}{\left(\left|a_{1}^{I M}\right|+\left|a_{2}^{I M}\right|\right) / 2}, \quad \hat{\mathbf{s}}=\frac{\mathbf{s}}{\left(\left|s_{1}^{I M}\right|+\left|s_{2}^{I M}\right|\right) / 2}
$$

These two vector metrics were then calculated at each critical location along the $x$-axis corresponding to a characteristic topographical feature as indicated in Fig. 3.

The shear stress distribution results were "normalised" by applying the same operation shown in Equation (9) to each data point. This normalised distribution was used to provide a graphical representation of the shear stress intensity and scattering in the form of an ellipse plot, as illustrated in Fig. 4. The ellipse equation was developed by identifying two additional scalar quantities $\bar{a}=\left(\hat{a}_{1}+\hat{a}_{2}\right) / 2$ and $\bar{s}=\left(\hat{s}_{1}+\hat{s}_{2}\right) / 2$, which were defined as the coordinates of the ellipse centre. The major and minor radii were defined as $r_{x}=\left(\hat{s}_{1}-\hat{s}_{2}\right) / 2$ and $r_{y}=\left(\hat{a}_{1}-\hat{a}_{2}\right) / 2$, so the ellipse equation was given by:

$$
\frac{(x-\bar{a})^{2}}{r_{x}^{2}}+\frac{(y-\bar{s})^{2}}{r_{y}^{2}}=1
$$

The ellipse encodes information about magnitude and spread of shear stresses (normalised intensity and scattering values) observed in the shear stress distribution. For the idealised skin model, the ellipse degenerates to a circle of radius 1 so it is easy to compare the non-circular ellipses of the anatomical models against these corresponding particular ellipses (i.e. circles). Any deviation from a circular shape along the $x$-direction indicates changes in scattering whilst any deviation along the $y$-direction indicates alterations of intensity. The ellipse plots offer a direct and visually simple means to illustrate how topographical features of the skin surface (as well as the underlying microstructure) transmit and induce shear stress in the viable epidermis.

\section{Results}

\subsection{Normal indentation}

The analysis of the shear stress distribution within the viable epidermis was performed by comparing, for each indenter size, the magnitude of shear stress experienced by the two skin models at equal total deflection, from the first point of contact. The plotted results are restricted to the region of interest of the skin models (see Fig. 2). Fig. 6 to Fig. 8 show the shear stress distribution at each interaction scale (i.e. depending on indenter size) with respect to node location in the $x$ direction (i.e. showing different shear stress values observed throughout the viable epidermis thickness - in the $y$ direction-at the same $x$ location). Each of these figures compare the results obtained from the idealised and anatomical models, under both high and low humidity conditions (implicitly modelled through two values of the Young's modulus of the stratum corneum). The geometry of the anatomical model with respect to its location in the $x y$ plane is shown for the identification of topographyinduced effects on shear stress distribution and magnitude.

The shear stress distribution computed for normal indentation shows a characteristic pattern when the skin is modelled as an idealised flat multi-layer material (Fig. 6). As expected, due to the symmetry of this model in normal indentation, the shear stress distribution ahead and behind the indenter are centrally symmetric. Directly under the indenter, the material is in a state of nearly pure compression so shear stresses are close to zero. 
Next to this low shear region, the shear stresses spike to reach their maximum (positive) and minimum (negative) values, identified as the components of the intensity vector indicated in Equation (7), at a distance no further than 0.5 times the indenter radius from the indenter centre. As expected, such a symmetry is not present in the simulations with the anatomical model, and the maximum and minimum shear stresses could be found at distances from 0.07 to 0.80 times the indenter radius from the indenter centre. The results showed different effects on shear stress distribution conditioned by the Young's modulus of the stratum corneum and the indenter size.

For the analysis of micro-indentation ( $R_{1}=0.1 \mathrm{~mm}$, Fig. 6), the shear stress distribution in the anatomical skin model showed a similar trend to that of the idealised one. With a low skin deflection $\delta=23 \mu \mathrm{m}$, stiffening of the stratum corneum resulted in a reduction of the shear stress intensity in the viable epidermis of $59 \%$ and an increase of propagation distance of $131 \%$ for the idealised model. Similarly, in the anatomical model, the shear stress intensity was reduced by $48 \%$ but the distance of propagation increased by $341 \%$. As a result of the multiasperity contact, the simulations with soft stratum corneum showed an increase of $24 \%$ in shear stress magnitude and a $16 \%$ larger shear stress propagation region. For the simulation with a stiff stratum corneum, these comparative values were $57 \%$ larger in intensity and $121 \%$ larger in propagation region.

Results of the simulations for indenter radius $R_{1}=0.25 \mathrm{~mm}$ and a skin deflection $\delta=50 \mu \mathrm{m}$ are presented in Fig. 7. For the idealised model, the shear stresses intensity calculated for $E_{S C}=370 \mathrm{MPa}$ was only $2 \%$ larger than that for $E_{S C}=0.6 \mathrm{MPa}$, but the region of shear stress propagation was $49 \%$ larger. Similar effects were observed in the anatomical model, with a small difference of $-5 \%$ in the shear stress intensity and region of shear stress propagation 32\% larger as the Young's modulus of the stratum corneum was increased. It was observed that for the anatomical model the shear stress intensity was $85 \%$ larger and the propagation region was $178 \%$ larger than for the idealised model when considering a soft stratum corneum ( $E_{S C}=0.6 \mathrm{MPa}$ ). Similarly, with a stiffer stratum corneum, the shear stress intensity was $71 \%$ larger and the propagation region showed an increase of $146 \%$.

In comparison with the results shown in Fig. 7, the simulations with $R_{1}=0.50 \mathrm{~mm}$ (Fig. 8) resulted in more than double the deflection (i.e. $\delta=120 \mu \mathrm{m}$ ). Significantly larger stresses were found near the contact area. It should be noted that despite the different levels of deformation imposed by the three indenter sizes, at distances further than $0.5 \mathrm{~mm}$ from the indenter centre, the shear stress distribution was within the same range observed for their smaller indenter counterparts. With the bigger indenter radius, the effects of stiffening of the stratum corneum was an amplification of the shear stress intensity and spread of the propagation region of $18 \%$ and $41 \%$ respectively, for the idealised model. Similar magnification of shear stress was observed for the anatomical model as the intensity of the shear stress was on average $20 \%$ larger with the stiffer stratum corneum. However, for that case, the increase in propagation region was only $2 \%$. When comparing the results obtained for the anatomical model to those of its idealised counterpart, with a soft stratum corneum, it was observed an increase of $163 \%$ in both shear stress intensity and propagation region. In the case of a stiffer outer layer $E_{S C}=370 \mathrm{MPa}$, the anatomical model exhibited an increase in the intensity of shear stresses of $169 \%$ and a shear stress propagation region $90 \%$ larger than the one observed in the idealised case.

\subsection{Sliding indentation}

It becomes more evident how the sliding motion and the value of the local coefficient of friction modify the distribution of shear stress in the viable epidermis. These results were first evaluated in the idealised skin model (Fig. 9) and then, compared to the shear stress distribution results of the anatomical model at different indenter locations (Fig. 11-Fig. 12 using the approach described in Section 2.4. The typical shear stress distributions of the idealised model simulations, as observed when the indenter is located at the mid-section of the region of interest,

With single asperity contact in the idealised skin model, the shear stress distribution was fairly consistent throughout the sliding motion, as the shear stress intensity and scattering vectors measured at three different locations had a mean standard deviation $0.68 \%$ in intensity and $2.06 \%$ in scattering. The typical shear stress distributions of the idealised model simulations sampled in the middle section of the region of interest are shown in Fig. 9. Given that at $\mu_{l}=0$ the maximum and minimum shear stresses are of the same magnitude (with a difference lower than $0.63 \%$ ), the results were normalised with respect to the frictionless simulation results between -1 and 1 instead of the more traditional normalisation between 0 and 1 . Using these values as a baseline, the effects of increasing the local coefficient of friction on the intensity of maximum (i.e. positive) and minimum (i.e. negative) shear stresses in the viable epidermis were quantified. 
The shear distribution in the viable epidermis was sensitive to the stiffness of the stratum corneum and the indenter radius but featured a similar qualitative trend across the six cases. As intuitively expected, an increase in the local coefficient of friction had the effect of shifting the zone of maximum shear intensity in the viable epidermis away from the indenter centre in the opposite direction of motion. As reported in 
Table 1, the range of normalised shear stresses (denoted by $\left(\hat{a}_{1}-\hat{a}_{2}\right) / 2$ ) increased by up to $54 \%$ in the idealised models with soft stratum corneum, but was maintained fairly constant (maximum increment of $1.3 \%$ was observed in the simulation with $R_{1}=0.10 \mathrm{~mm}$ and $\mu_{l}=0.3$ ) for the simulations featuring a stiff stratum corneum. For both values of stratum corneum Young's modulus, the level of scattering, in contrast, is conditioned by indenter size and skin deflection. This dependency was identified through two non-linear regressions: $s_{1}-s_{2}=1.213 R_{1}^{-0.498}\left(\mathrm{R}^{2}=0.877\right)$ for $E_{S C}=0.6 \mathrm{MPa}$ and $s_{1}-s_{2}=1.299 R_{1}^{-0.672}$ for $E_{S C}=370 \mathrm{MPa}$ $\left(\mathrm{R}^{2}=0.941\right.$ ) (see Fig. 10).

Using the visualisation approach described in Section 2.4, the sensitivity of shear stress intensity and scattering vectors ( $\mathbf{a}$ and $\mathbf{s}$, respectively) to indenter size, local coefficient of friction and Young's modulus of the stratum corneum is depicted in Fig. 11 and Fig. 12. This sensitivity was assessed within the viable epidermis at each location immediately underneath each of the 11 topographical features of the skin identified by the letters $A$ to $L$ (Fig. 3). Each of the idealised cases show a circle of normalised radius equal to 1 which indicates the scale of the anatomical normalised ellipse plot.

By inspecting Fig. 11 and Fig. 12, it is clearly apparent that each geometrical feature of the skin surface is correlated with variations in shear stress intensity and scattering for both soft and stiff stratum corneum. Like the local coefficient of friction, the indenter size is also an important contributing factor. This was particularly obvious for the topographic features $[A, B, F, H, K, L]$ below the mean height of the skin surface where the indentation depth was relatively low with the small indenter and significantly larger with the bigger indenters, resulting in amplification of the shear stress intensity and scattering metrics. Variation of the elastic modulus of the stratum corneum leads to significant changes in the shear stress distribution. The computational analyses featuring a stiff stratum corneum highlighted higher shear stress intensity and lower scattering than when considering the minimum value for the elastic modulus. For smaller indenter radius/indentation depth, the shear stress intensity and scattering showed mixed amplification and reduction, with no obvious correlation to the height of topographic features.

For comparing the mechanical response of the anatomical models to that of the idealised models, and in order to extract a general trend from the finite element analyses without considering every individual topographic feature of the skin surface (and underlying microstructure), the results graphically presented in Fig. 11 and Fig. 12 were averaged over the 11 topographical features. Results were compared in terms of shear stress intensity and scattering ratios, calculated as the average of the ellipse radii ( $r_{y}$ for intensity and $r_{x}$ for scattering). These average ratios are listed in Table 2 . The intensity ratio indicates the mean amplification of the maximum shear stresses observed in the viable epidermis with respect to the idealised case, and the scattering ratio reflects how further these stresses propagate from the region of contact. In order to avoid the geometrical effects of the flat surfaces outside the region of interest, only topographic features D-J were considered for this analysis.

These results showed that for the particular skin topography and internal microstructure of the anatomical model considered in the current study, the intensity of shear stress was on average 2.29 larger than for the equivalent idealised case. These results showed no direct correlation with local coefficient of friction nor indenter radius. For the softer stratum corneum $\left(E_{S C}=0.6 \mathrm{MPa}\right)$, the intensity ratio was consistently higher in frictionless conditions, for each indenter radius. The frictionless intensity ratios varied from 2.52 to 3.36 , and they ranged between 1.64 and 2.54 for the cases where $\mu_{l}>0$. The scattering ratio ranged between 2.37 and 2.73 for the frictionless case, but for the non-frictionless cases it was observed that the scattering ratio was more sensitive to the indenter size as the coefficient of friction increased: for $\mu_{l}=0.1$ the scattering ratio varies from 1.87 to 1.94 , while for $\mu_{l}=0.3$ the variation goes from 1.80 to 2.86 .

For the stiffer stratum corneum $\left(E_{S C}=370 \mathrm{MPa}\right.$ ), the intensity and scattering ratio showed no apparent correlation with the coefficient of friction between the indenter and the stratum corneum. The intensity ratio varied from 1.96 to 2.88 , and the scattering ratio from 1.58 to 2.22 . The intensity ratio tends to increase with larger indenter size while scattering decreases in these conditions. 


\section{Discussion}

When the skin interacts with external objects through contact interaction, the risk of developing skin injuries depends on many factors including not only the type and magnitude of loads, the microstructural/mechanical properties of the skin and contacting material, but also the tribo-physics of these surfaces. These properties are dynamic and sensitive to environmental conditions (e.g. softening of the stratum corneum with increased relative humidity and/or temperature) and, as a result, any of their variations can trigger a cascade of physical processes that can drastically alter the characteristics of the physical system across several spatial scales. For example, higher humidity levels soften the stratum corneum which results in swelling of corneocytes, larger deformation of the skin surface and therefore larger contact areas. The capillary forces between the skin and contacting materials increase the contribution of adhesion to the apparent (or macroscopic) coefficient of friction between the contacting parts (especially if the contacting material is hydrophilic) [25]. This frictionhumidity dependence is central to the notion of skin microclimate [5] and the study of mechanically-induced skin injuries, specifically skin tears, pressure ulcers and friction blisters, because higher friction leads to higher interfacial shear while excessive temperature in combination with plasticisation of the stratum corneum are known to compromise skin integrity $[4,5]$.

In most experimental studies of skin tribology and most of the numerical models of skin available in the literature, the skin is assumed to feature a flat surface where the deformation-induced component of skin friction is neglected. This assumption is often justified by invoking the plasticising effect of water on the stratum corneum [25] as explained above. In these conditions, it is thought that deformations of skin surface asperities are dominated by adhesive forces in term of their relative contribution to friction [25]. This assumption was recently called into question in a computational study [24] which demonstrated the significant effect of skin micro-asperities and internal microstructure on the deformation-induced component of skin friction. It is therefore legitimate to investigate the role that skin microrelief and internal microstructure could play on shear stress transmission and propagation within the living cellular layers of the skin.

It is reasonable to assume that mechanical damage to the skin in general, and viable epidermis in particular, will occur as soon as critical shear stress levels are reached. Friction blisters occur in the viable epidermis as a result of surface shear loads, and while pressure ulcers are related to compression-induced ischaemia and reperfusion of capillary flow, it is likely that excessive shear stresses experienced by living cells of the viable epidermis contribute to their mechanical damage and, subsequently to their death as evidenced for muscle cells [21]. In turn, degradation of the supporting structural and biological constituents of the viable epidermis will compromise skin mechanical integrity and biological functions, thus aggravating the likelihood of developing deeper tissue injuries (i.e. pressure sores) [12].

In the last decade, significant progress has been made on developing sensors to measure interfacial shear stresses on the skin of patients with low mobility (i.e. bed-or wheel chair-ridden patients) as well as for prosthetics wearers where optimising comfort at the stump-prosthetic interface and preventing pressure ulcers is critical $[17,48,49]$. These experimental techniques and tools offer vast opportunities to be complemented by computational studies to gain a more mechanistic understanding of the interplay between the physical factors that contribute to primary skin injuries and that can ultimately evolve into more serious conditions such as chronic wounds $[5,9,50]$.

To date, one of the main limitations of existing computational models is the oversimplification (i.e. idealisation) of the geometry of the skin layers. Only few studies have included structural information about the geometry of the skin surface and/or its internal sub-layers $[23,24,51]$. The current authors have been exploring this very topic by investigating the implications of the geometrical and mechanical interplay between skin surface topography and internal microstructure for different applications such as skin friction [24] or the emergence of micro-wrinkles induced by the structural characteristics of skin microrelief. Like the present work, these studies have highlighted the significant role of these microstructural and mechanical characteristics on the mechanics of skin. Here, it was demonstrated how accounting for those properties results in, not only drastically different shear stress distribution in the viable epidermis, but also in magnification of their intensity. As established in the present study, in that particular idealised case, the shear stress distribution in the viable epidermis is fairly predictable in the case of normal indentation (Fig. 6-Fig. 8): two regions of high shear stress magnitude are observed ahead and behind the centre of the indenter, but these shear stresses remain localised to the area of contact and do not spread much further, sidewise or deeper into the epidermis. 
This shear stress distribution pattern is expected to be observed also with higher coefficients of friction given the symmetry of the contacting geometries although the maximum shear stress values might increase, as observed in the idealised sliding contact simulations. For the anatomical model, shear stresses spike at each contact area corresponding to a micro-asperity, and also exhibit larger magnitude than those observed in the idealised skin model. Furthermore, these stresses propagate further away from the area of contact, mostly affecting the areas around the skin furrows.

Similar effects were observed in the sliding contact simulations. In idealised conditions, the pattern with two regions of high shear stress magnitude, ahead and behind the indenter, is also observed. With higher local coefficient friction, the pattern is shifted to the opposite direction of the indenter motion and the shear stresses ahead the indenter have higher intensity than those behind. Although the pattern characteristics (i.e. described by its intensity and scattering vectors, as well as the shift metric) are fairly predictable, high coefficient of friction and large indentation can disrupt the patterns as it was observed in the simulation with the parameters [ $E_{S C}=0.6 \mathrm{MPa}, R_{1}=0.5 \mathrm{~mm}, \mu_{l}=0.3$ ] in Fig. 9. In the idealised simulations, the shear stress distribution is less sensitive to changes to the local coefficient of friction if the stratum corneum is on its dryer (stiffer) state. In the anatomical model, the shear stress distribution is also dependent on the localised micro-asperity contacts, but how much the shear stresses propagate away from the area of contact is highly dependent upon the location of the indenter over the skin topography, indenter size/depth of indentation, stiffness of the stratum corneum and local coefficient of frictions (or interface conditions).

As expected, under normal indentation, a stiffer stratum corneum ( $E_{S C}=370 \mathrm{MPa}$ ) leads to shear stresses that are less localised than for a softer one. However, under sliding indenting conditions the opposite effect was observed: as shown in Fig. $\mathbf{1 1}$ and Fig. 12, the scattering metric used as a measure of shear stress propagation is lower for the simulations with stiffer outer layer than those with the soft one (see also Table 2). This is because the deformation mechanism is different in these two simulations. Under normal indentation, the skin directly underneath the indenter is in a state of nearly pure compression while the skin surface surrounding that zone bends by the action of the indenter, also leading to tensile stresses. With a soft stratum corneum, most deformations are localised to the nearby furrows [23]. Under sliding contact, the skin is stretched behind the indenter and pushed/compressed ahead of it. These deformations are conditioned by the level of drag (directly correlated to the local coefficient of friction) and the resistance provided by the skin micro-asperities under the form of geometrical constraints. With a soft stratum corneum, greater drag results in larger contact area, so pressure is more widely distributed and the intensity of the shear stresses for $\mu_{l}>0$ are lower than those observed in frictionless conditions. As in our model the lateral boundaries are free to move, the stiffer stratum corneum displaces the layers underneath under the action of the indenter rather than deforming and transmitting this deformation to the viable epidermis, resulting in lower scattering than for the sliding simulations with a soft stratum corneum.

The results of this study show that the intensity of the shear stresses in the anatomical model are significantly higher than the shear stresses observed in the idealised skin model. Although the intensity vector components at some topographic features were up to 6.7 times larger than their idealised model counterpart (e.g. $S_{2}$ for topographic feature $\mathrm{E}$ for the simulation parameters $\left[E_{S C}=370 \mathrm{MPa}, R_{1}=0.25 \mathrm{~mm}\right.$ ] with $\mu_{l}=0.0$, and $s_{1}$ for same parameters with $\mu_{1}=0.3$ ), the mean amplification (i.e. intensity ratio) of the shear stresses was 2.29 and 2.38 for the simulations with $E_{S C}=0.6 \mathrm{MPa}$ and $E_{S C}=370 \mathrm{MPa}$, respectively.

By maintaining a constant indentation depth with respect to the mean skin surface, it would be expected that the shear stresses would be related to the height of the topographic features with respect to the mean surface. However, even the topographic features $[F, J, L]$, located within $\pm 0.007 \mathrm{~mm}$ of the mean surface, show great amplification on the intensity and scattering metrics.

The results of this study are intrinsically linked to the geometry of the skin sample used for the anatomical model, so different results would be expected for skin samples with different surface roughness, internal microstructure and mechanical properties. Notwithstanding this fact, Fig. 11 and Fig. 12 illustrate how the risk of damage initiation (correlated with high shear stress) varies according to the contact conditions at specific topographic and internal microstructural features of the skin and how far an "idealised" distribution of shear stress might be from that naturally occurring in real in vivo conditions. 
To date, experimental studies have analysed interfacial shear stress as a surrogate in vivo measure of the risk of developing skin injuries [16-18]. Nevertheless, these studies do not provide a quantitative insight into how these stresses are transmitted from the surface to the deeper layers of the skin. Moreover, interfacial shear measurements ignore the sub-millimetric texture of human skin and associated deformations so that shear measurements are analysed by considering the apparent areas rather than real areas. To the best of our knowledge, only Sopher and Gefen [9] have attempted to use a computational model to estimate the risk of skin injury, using the interfacial shear stress of $70 \mathrm{kPa}$ measured by Goldstein and Sanders [22] as shear stress threshold for potential damage within the skin.

The study presented in this paper was restricted to a 2D setting using a plane-strain and isotropic neo-Hookean hyperelastic constitutive formulation. These structural and constitutive aspects represent certain limitations of the current model as, naturally, the skin is a geometrically and materially complex 3D structure featuring nonlinear elastic and inelastic phases. The interplay of these characteristics with non-uniform boundary and loading conditions induce complex behaviour. However, like in our previous studies [23,24], our modelling approach is to develop mechanistic models as simple as possible (but, to paraphrase Albert Einstein, "not simpler") with a minimum (but sufficient) number of parameters so that plausible structural mechanisms interacting with constitutive material aspects of the skin could be unveiled and, by so doing, allow us to advance our understanding of skin biomechanics and formulate new hypotheses.

In the study presented in this paper, our goal was to gain a quantitative insight into shear load transmission from the skin surface to the deeper layers and asses the role of the skin microstructure (geometrical and material effects). For example, accounting for viscoelasticity-a well-known property of the skin-would add a time dependency to our results and, possibly, modulation of stress and strain magnitude. Making our model "materially" more sophisticated would not add any substantial information that would address our original research question. These restricting assumptions could also be viewed as strengths as they facilitate interpretation of results by isolating and potentially decoupling the effects of certain parameters such as mechanical properties or geometry of skin layers.

We strongly believe that using basic models that can later be extended and accommodate additional complexity is a sensible approach to understand, step by step, the contribution of different parameters to the physical response of the skin during contact interactions. In a 3D setting, by considering or not the fibrous-and therefore directional-nature of the dermis due to the presence of an intricate network of collagen fibres [52-54], complex 3D anisotropic structural effects arising from the anatomical skin microstructure would be observed [23]. Future studies should also consider the existence of residual strains in the dermal layer in vivo [54, 55] as these are important factors that conditional the overall mechanical response of the skin. 


\section{Conclusion}

Despite much attention in experimentally and computationally characterising shear stress at the surface of the skin in relation to skin injuries and pressure ulcers, very little efforts have been made to develop methodologies to gain a more accurate and mechanistic understanding of how shear stresses are induced and how they propagate from the skin surface to the deeper layers where they are likely to mechanically stress living cells. Ultimately, excessive stress or strain can lead to cell damage and death which, at a meso/macroscopic level translates into tissue damage and loss of structural integrity.

The present computational study has demonstrated, like in recent studies of Leyva-Mendivil et al. [23, 24], that the skin topography and microstructure (i.e. geometry and mechanical properties of its layers) can play a major role on its mechanical response, particularly when combined with variations of other system parameters such as the local (i.e. microscopic) coefficient of friction or the size of the indenting object.

Results showed that the multi-asperity contact induced by the skin topographic features in combination with a non-rectilinear (i.e. non-idealised) geometry of the skin layers lead to levels of shear stresses within the living epidermis much higher than those produced in the geometrically-idealised case. These findings have significant implications for the design and analyses of finite element studies aiming at describing the tribology of skin, relevant to a wide range of applications from pressure ulcers, friction-induced burns (e.g. like those occurring during airbag deployment) to personal care products and ergonomics. Most of published experimental and computational studies measuring or computing shear stress either focus on those observed at the external surface of the skin (typically assumed to be flat in computational models) or at internal boundaries between skin layers or skin and muscles (often assumed to feature idealised geometries or anatomical geometries ignoring microstructural details). Our study clearly demonstrated that this is insufficient if one seeks to gain a truly mechanistic understanding of the mechanical behaviour of the epidermis in response to contact solicitations of the skin surface. It is the authors 'opinion that the road to develop an in-depth understanding of the mechanobiological conditions leading to the occurrence and evolution of skin damage in relation to biotribological interactions can only be taken within an integrated multiscale approach.

The results, numerical tools and methodologies developed in this study are preliminary stepping stones that will ultimately be completed by/complement experimental protocols so that engineers designing products intended to interact with the skin could do so within the framework of a unified physics-based integrative environment.

\section{Acknowledgements}

This work was partially supported by the Mexican National Council of Science and Technology (CONACYT) [311260] and the Engineering and Physical Sciences Research Council [EP/F034296/1]. Their financial support is gratefully acknowledged. The authors would also like to thank Dr. David-John O'Callaghan of Procter \& Gamble for many fruitful discussions.

\section{Conflicts of interest}

None to declare. 


\section{References}

[1] T. Burns, S. Breathnach, N. Cox, C. Griffiths, Rook's Textbook of Dermatology, 7th ed., Blackwell Science, Oxford, 2004.

[2] F.M. Hendriks, Mechanical behaviour of human epidermal and dermal layers in vivo, 2005.

[3] L.C. Gerhardt, V. Strässle, a. Lenz, N.D. Spencer, S. Derler, Influence of epidermal hydration on the friction of human skin against textiles, Journal of the Royal Society, Interface / the Royal Society 5(28) (2008) 1317-28.

[4] R.R. Warner, K.J. Stone, Y.L. Boissy, Hydration disrupts human stratum corneum ultrastructure, Journal of Investigative Dermatology 120(2) (2003) 275-284.

[5] A. Gefen, How do microclimate factors affect the risk for superficial pressure ulcers: A mathematical modeling study, Journal of Tissue Viability 20(3) (2011) 81-88.

[6] W. Zhong, M.M.Q. Xing, N. Pan, H.I. Maibach, Textiles and Human Skin, Microclimate, Cutaneous Reactions: An Overview, Cutaneous and Ocular Toxicology 25(1) (2006) 23-39.

[7] C.P. Hendriks, S.E. Franklin, Influence of Surface Roughness, Material and Climate Conditions on the Friction of Human Skin, Tribology Letters 37(2) (2010) 361-373.

[8] S. Derler, L.C. Gerhardt, Tribology of skin: Review and analysis of experimental results for the friction coefficient of human skin, 2012, pp. 1-27.

[9] R. Sopher, A. Gefen, Effects of skin wrinkles, age and wetness on mechanical loads in the stratum corneum as related to skin lesions, Medical \& Biological Engineering \& Computing 49(1) (2011) 97-105.

[10] H. Shimizu, Shimizu's Textbook of Dermatology, 1st ed., Hokkaido University Press/Nakayama Shoten, Hokkaido, Japan, 2007.

[11] Flexion Reflex Pathways, in: D.A. Purves, George J; Fitzpatrick, David; Katz, Lawrence C; LaMantia, AnthonySamuel; McNamara, James O; Williams., S Mark (Ed.), Neuroscience, Sunderland (MA): Sinauer Associates2001. [12] C.V. Bouten, C.W. Oomens, F.P. Baaijens, D.L. Bader, The etiology of pressure ulcers: Skin deep or muscle bound?, Arch. Phys. Med. Rehabil. 84(4) (2003) 616-619.

[13] E. Linder-Ganz, A. Gefen, Mechanical compression-induced pressure sores in rat hindlimb: muscle stiffness, histology, and computational models, Journal of Applied Physiology 96(6) (2004) 2034-2049.

[14] E. Linder-Ganz, N. Shabshin, A. Gefen, Biomechanical Analysis of a Serious Pressure Ulcer Case in a RealWorld Scenario, Proceedings of the Asme Summer Bioengineering Conference - 2009, Pt a and B (2009) 669670.

[15] F. Hashmi, B.S. Richards, S. Forghany, A.L. Hatton, C.J. Nester, The formation of friction blisters on the foot: The development of a laboratory-based blister creation model, Skin Research and Technology 19 (2013) 479489.

[16] E. Call, J. Pedersen, B. Bill, J. Black, P. Alves, C.T. Brindle, C. Dealey, N. Santamaria, M. Clark, Enhancing pressure ulcer prevention using wound dressings: what are the modes of action?, Int Wound J 12(4) (2015) 408413.

[17] P. Laszczak, L. Jiang, D.L. Bader, D. Moser, S. Zahedi, Development and validation of a 3D-printed interfacial stress sensor for prosthetic applications, Med Eng Phys 37(1) (2015) 132-137.

[18] M. Yavuz, American Society of Biomechanics Clinical Biomechanics Award 2012: Plantar shear stress distributions in diabetic patients with and without neuropathy, Clin Biomech 29(2) (2014) 223-229.

[19] J.J. Knapik, K.L. Reynolds, K.L. Duplantis, B.H. Jones, Friction Blisters, Sports Medicine 20(3) (1995) 136-147. [20] D.L. Bader, C.V.C. Bouten, D. Colin, Pressure Ulcer Research: Current and Future Perspectives, Springer Berlin Heidelberg2005.

[21] Y. Wu, D.W.J. van der Schaft, F.P. Baaijens, C.W.J. Oomens, Cell death induced by mechanical compression on engineered muscle results from a gradual physiological mechanism, Journal of Biomechanics 49(7) (2016) 1071-1077.

[22] B. Goldstein, J. Sanders, Skin Response to Repetitive Mechanical Stress : a New Experimental Model in Pig, Arch. Phys. Med. Rehabil. 79(3) (1998) 265-272.

[23] M.F. Leyva-Mendivil, A. Page, N.W. Bressloff, G. Limbert, A mechanistic insight into the mechanical role of the stratum corneum during stretching and compression of the skin, Journal of the Mechanical Behavior of Biomedical Materials 49 (2015) 197-219.

[24] M.F. Leyva-Mendivil, J. Lengiewicz, A. Page, N.W. Bressloff, G. Limbert, Skin Microstructure is a Key Contributor to Its Friction Behaviour, Tribology Letters 65(1) (2017).

[25] M.J. Adams, B.J. Briscoe, S.A. Johnson, Friction and lubrication of human skin, Tribology Letters 26(3) (2007) 239-253.

[26] J.R. Barber, Plane Strain and Plane Stress, Elasticity, Springer Netherlands. Kluwer Academic Publishers, London, 2002, pp. pp 33-40. 
[27] A.R. Karduna, H.R. Halperin, F.C.P. Yin, Experimental and numerical analyses of indentation in finite-sized isotropic and anisotropic rubber-like materials, Annals of Biomedical Engineering 25(6) (1997) 1009-1016.

[28] J. Korelc, Multi-language and multi-environment generation of nonlinear finite element codes, Engineering with Computers 18(4) (2002) 312-327.

[29] J. Korelc, P. Wriggers, Automation of Finite Element Methods, 1 ed., Springer International Publishing 2016. [30] G.A. Holzapfel, Nonlinear Solid Mechanics. A Continuum Approach for Engineering, John Wiley \& Sons, Chichester, UK, 2000.

[31] N.T. Clancy, G.E. Nilsson, C.D. Anderson, M.J. Leahy, A new device for assessing changes in skin viscoelasticity using indentation and optical measurement, Skin Research and Technology 16(2) (2010) 210-228. [32] M. Geerligs, L. van Breemen, G. Peters, P. Ackermans, F. Baaijens, C. Oomens, In vitro indentation to determine the mechanical properties of epidermis, Journal of Biomechanics 44(6) (2011) 1176-1181.

[33] J.T. livarinen, R.K. Korhonen, P. Julkunen, J.S. Jurvelin, Experimental and computational analysis of soft tissue stiffness in forearm using a manual indentation device, Med Eng Phys 33(10) (2011) 1245-1253.

[34] K.S. Wu, W.W. van Osdol, R.H. Dauskardt, Mechanical properties of human stratum corneum: effects of temperature, hydration, and chemical treatment, Biomaterials 27(5) (2006) 785-95.

[35] J. Serup, G.B.E. Jemec, G. Grove, Handbook of Non-Invasive Methods and the Skin, Boca Raton FL: CRC Press1995.

[36] A. Delalleau, G. Josse, J.-M. Lagarde, H. Zahouani, J.-M. Bergheau, Characterization of the mechanical properties of skin by inverse analysis combined with the indentation test, Journal of Biomechanics 39(9) (2006) 1603-1610.

[37] Y. Gu, J. Li, X. Ren, M.J. Lake, Y. Zeng, Heel skin stiffness effect on the hind foot biomechanics during heel strike, Skin Research and Technology 16(3) (2010) 291-296.

[38] F. Khatyr, C. Imberdis, D. Varchon, J.-M. Lagarde, G. Josse, Measurement of the mechanical properties of the skin using the suction test, Skin Research and Technology 12(1) (2006) 24-31.

[39] C. Pailler-Mattei, S. Bec, H. Zahouani, In vivo measurements of the elastic mechanical properties of human skin by indentation tests, Med Eng Phys 30(5) (2008) 599-606.

[40] J.L. Lévêque, B. Audoly, Influence of Stratum Corneum on the entire skin mechanical properties, as predicted by a computational skin model, Skin Research and Technology 19(1) (2013) 42-46.

[41] N. Magnenat-Thalmann, P. Kalra, J. Luc Leveque, R. Bazin, D. Batisse, B. Querleux, A computational skin model: fold and wrinkle formation, IEEE Transactions on Information Technology in Biomedicine 6(4) (2002) 317323.

[42] J. Lengiewicz, J. Korelc, S. Stupkiewicz, Automation of finite element formulations for large deformation contact problems, Int J Numer Meth Eng 85(10) (2011) 1252-1279.

[43] N.M. Newmark, A Method of Computation for Structural Dynamics Journal of the Engineering Mechanics Division 85(3) (1959) 67-94.

[44] G. Stachowiak, A.W. Batchelor, Engineering Tribology, Butterworth-Heinemann, 2013.

[45] M.J. Puttock, E.G. Thwaite, Elastic Compression of Spheres and Cylinders at Point and Line Contact, National Standards Laboratory Technical Paper 25(25) (1969).

[46] K.L. Johnson, Contact Mechanics, Cambridge University Press, Cambridge, UK., 1985.

[47] I.M. Sobol, On the distribution of points in a cube and the approximate evaluation of integrals, USSR Computational Mathematics and Mathematical Physics 7(4) (1967) 86-112.

[48] L.A. de Wert, D.L. Bader, C.W.J. Oomens, L. Schoonhoven, M. Poeze, N.D. Bouvy, A new method to evaluate the effects of shear on the skin, Wound Repair and Regeneration 23(6) (2015) 885-890.

[49] P. Laszczak, M. McGrath, J. Tang, J. Gao, L. Jiang, D.L. Bader, D. Moser, S. Zahedi, A pressure and shear sensor system for stress measurement at lower limb residuum/socket interface, Med Eng Phys 38(7) (2016) 695700.

[50] C.W.J. Oomens, W. Zenhorst, M. Broek, B. Hemmes, M. Poeze, P.R.G. Brink, D.L. Bader, A numerical study to analyse the risk for pressure ulcer development on a spine board, Clin Biomech 28(7) (2013) 736-742.

[51] J. van Kuilenburg, M.A. Masen, E. van der Heide, The role of the skin microrelief in the contact behaviour of human skin: Contact between the human finger and regular surface textures, Tribology International 65 (2013) 81-90.

[52] Y. Lanir, Y.C. Fung, Two-Dimensional Mechanical Properties of Rabbit Skin-II: Experimental Results, Journal of Biomechanics 7 (1972) 171-182.

[53] A. Ní Annaidh, K. Bruyère, M. Destrade, M.D. Gilchrist, C. Maurini, M. Otténio, G. Saccomandi, Automated estimation of collagen fibre dispersion in the dermis and its contribution to the anisotropic behaviour of skin, Annals of Biomedical Engineering 40(8) (2012) 1666-1678. 
[54] A. Ní Annaidh, K. Bruyère, M. Destrade, M.D. Gilchrist, M. Otténio, Characterization of the anisotropic mechanical properties of excised human skin, Journal of the Mechanical Behavior of Biomedical Materials 5(1) (2011) 139-148.

[55] K. Langer, Zur Anatomie und Physiologie der Haut. Über die Spaltbarkeit der Cutis, Sitzungsbericht der Mathematisch-naturwissenschaftlichen Classe der Wiener Kaiserlichen Academie der Wissenschaften Abt 44 (1861). 
Table 1 Results of the sliding simulations of the idealised skin model. Maximum and minimum shear stresses are normalised with respect to the cases where $\mu_{l}=0$, scattering vector components, coefficients of the shear stress distribution equation estimated form the location and magnitude of the normalised maximum and minimum shear stresses.

\begin{tabular}{|c|c|c|c|c|c|c|c|}
\hline$E_{S C}$ & $\begin{array}{c}R_{1} \\
{[\mathrm{~mm}]}\end{array}$ & $\mu_{l}$ & $\begin{array}{c}\text { Normalised } \\
\text { maximum } \\
\text { shear stress } \hat{a}_{1}\end{array}$ & $\begin{array}{c}\text { Normalised } \\
\text { minimum } \\
\text { shear stress } \\
\hat{a}_{2} \\
\end{array}$ & $\frac{\hat{a}_{1}-\hat{a}_{2}}{2}$ & $\begin{array}{c}S_{1} \\
{\left[R_{1}\right]}\end{array}$ & $\begin{array}{c}S_{2} \\
{\left[R_{1}\right]}\end{array}$ \\
\hline 0.6 & 0.10 & 0.0 & 1.000 & -1.000 & 1.000 & 0.324 & -0.657 \\
\hline 0.6 & 0.10 & 0.1 & 1.384 & -1.185 & 1.284 & 0.484 & -0.718 \\
\hline 0.6 & 0.10 & 0.2 & 1.494 & -1.074 & 1.284 & 0.554 & -0.680 \\
\hline 0.6 & 0.10 & 0.3 & 1.586 & -0.970 & 1.278 & 0.620 & -0.647 \\
\hline 0.6 & 0.25 & 0.0 & 1.000 & -1.000 & 1.000 & 0.316 & -0.649 \\
\hline 0.6 & 0.25 & 0.1 & 1.412 & -1.198 & 1.305 & 0.484 & -0.711 \\
\hline 0.6 & 0.25 & 0.2 & 1.513 & -1.089 & 1.301 & 0.560 & -0.635 \\
\hline 0.6 & 0.25 & 0.3 & 1.624 & -0.979 & 1.302 & 0.629 & -0.594 \\
\hline 0.6 & 0.50 & 0.0 & 1.000 & -1.000 & 1.000 & 0.320 & -0.603 \\
\hline 0.6 & 0.50 & 0.1 & 1.558 & -1.335 & 1.446 & 0.544 & -0.761 \\
\hline 0.6 & 0.50 & 0.2 & 1.668 & -1.226 & 1.447 & 0.606 & -0.706 \\
\hline 0.6 & 0.50 & 0.3 & 1.798 & -1.295 & 1.547 & 0.681 & -0.713 \\
\hline 370 & 0.10 & 0.0 & 1.000 & -1.000 & 1.000 & 0.323 & -0.559 \\
\hline 370 & 0.10 & 0.1 & 1.016 & -0.992 & 1.004 & 0.360 & -0.554 \\
\hline 370 & 0.10 & 0.2 & 1.042 & -0.972 & 1.007 & 0.401 & -0.551 \\
\hline 370 & 0.10 & 0.3 & 1.063 & -0.963 & 1.013 & 0.446 & -0.622 \\
\hline 370 & 0.25 & 0.0 & 1.000 & -1.000 & 1.000 & 0.317 & -0.612 \\
\hline 370 & 0.25 & 0.1 & 1.030 & -0.975 & 1.002 & 0.359 & -0.580 \\
\hline 370 & 0.25 & 0.2 & 1.063 & -0.949 & 1.006 & 0.412 & -0.505 \\
\hline 370 & 0.25 & 0.3 & 1.096 & -0.929 & 1.012 & 0.455 & -0.493 \\
\hline 370 & 0.50 & 0.0 & 1.000 & -1.000 & 1.000 & 0.320 & -0.555 \\
\hline 370 & 0.50 & 0.1 & 1.048 & -0.956 & 1.002 & 0.374 & -0.611 \\
\hline 370 & 0.50 & 0.2 & 1.097 & -0.916 & 1.006 & 0.417 & -0.569 \\
\hline 370 & 0.50 & 0.3 & 1.150 & -0.874 & 1.012 & 0.466 & -0.520 \\
\hline
\end{tabular}


Table 2 Intensity and scattering ratios of the anatomical model simulations with respect to their corresponding idealised counterparts.

\begin{tabular}{cccccc}
\hline \hline \multirow{2}{*}{$R_{1}$} & \multirow{2}{c}{ Intensity ratio } & \multicolumn{2}{c}{ Scattering ratio } \\
\cline { 3 - 6 }$[\mathrm{mm}]$ & $\mu_{1}$ & $E_{S C}=0.6 \mathrm{MPa}$ & $E_{S C}=370 \mathrm{MPa}$ & $E_{S C}=0.6 \mathrm{MPa}$ & $E_{S C}=370 \mathrm{MPa}$ \\
\hline \hline 0.1 & 0 & 2.52 & 2.1 & 2.73 & 2.22 \\
0.1 & 0.1 & 1.96 & 1.96 & 1.94 & 1.89 \\
0.1 & 0.2 & 1.85 & 2.33 & 2.43 & 2.08 \\
0.1 & 0.3 & 1.99 & 2.15 & 2.86 & 2.22 \\
\hline 0.25 & 0 & 2.79 & 2.63 & 2.68 & 1.97 \\
0.25 & 0.1 & 2.1 & 1.97 & 1.92 & 1.98 \\
0.25 & 0.2 & 2.09 & 2.12 & 2.2 & 2.22 \\
0.25 & 0.3 & 2.13 & 2.43 & 2.57 & 2.08 \\
\hline 0.5 & 0 & 3.36 & 2.64 & 2.37 & 1.66 \\
0.5 & 0.1 & 2.45 & 2.88 & 1.87 & 1.63 \\
0.5 & 0.2 & 2.54 & 2.55 & 1.89 & 1.68 \\
0.5 & 0.3 & 1.65 & 2.82 & 1.8 & 1.58 \\
\hline \multicolumn{2}{c}{ Average } & $\mathbf{2 . 2 9}$ & $\mathbf{2 . 3 8}$ & $\mathbf{2 . 2 7}$ & $\mathbf{1 . 9 3}$ \\
\hline \hline
\end{tabular}




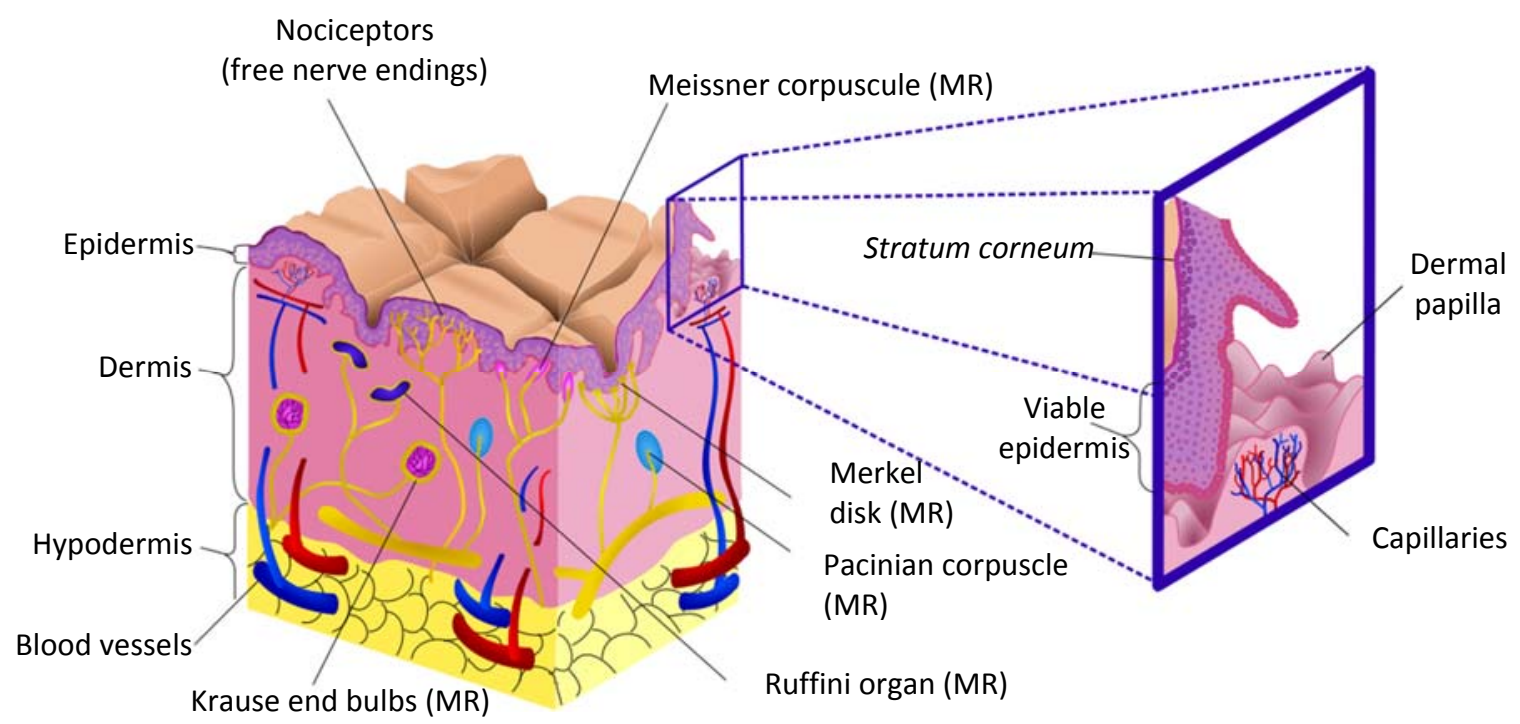

Fig. 1 Schematic representation of the anatomical structure of human skin including its sensory receptors [mechanoreceptors (MR) and nociceptors].

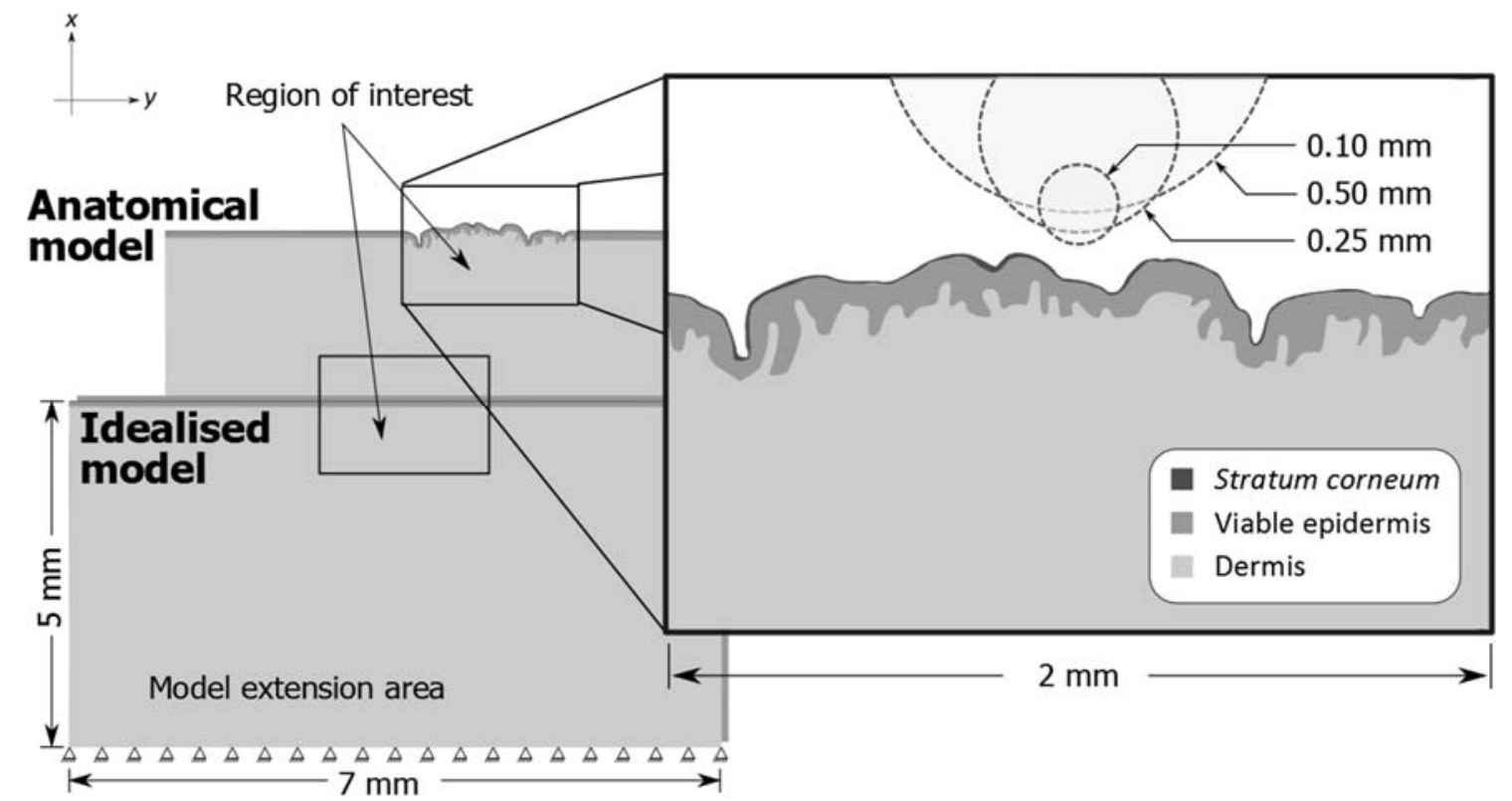

Fig. 2. Skin models. The anatomical (top) and idealised (bottom) skin models were appropriately dimensioned to avoid boundary effects in the finite element analyses [27]. A zoomed-in view of the detailed plane-strain mesh of the anatomicallybased portion of the skin model is provided as well as the dimensions of the region of interest. Boundary conditions are also indicated on the figure. 


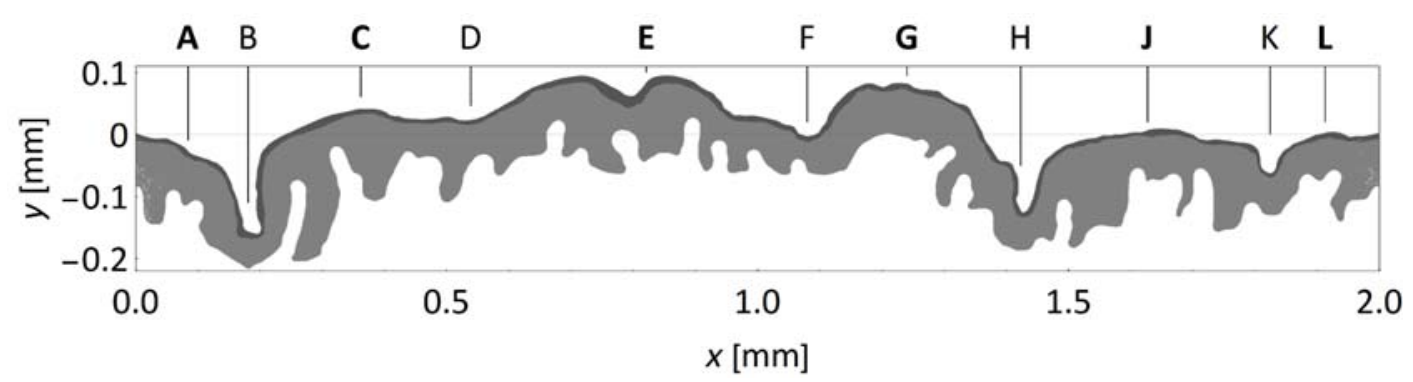

Fig. 3 Identification of characteristic topographic features of the skin surface for the anatomical skin model (crests are indicated in bold characters).

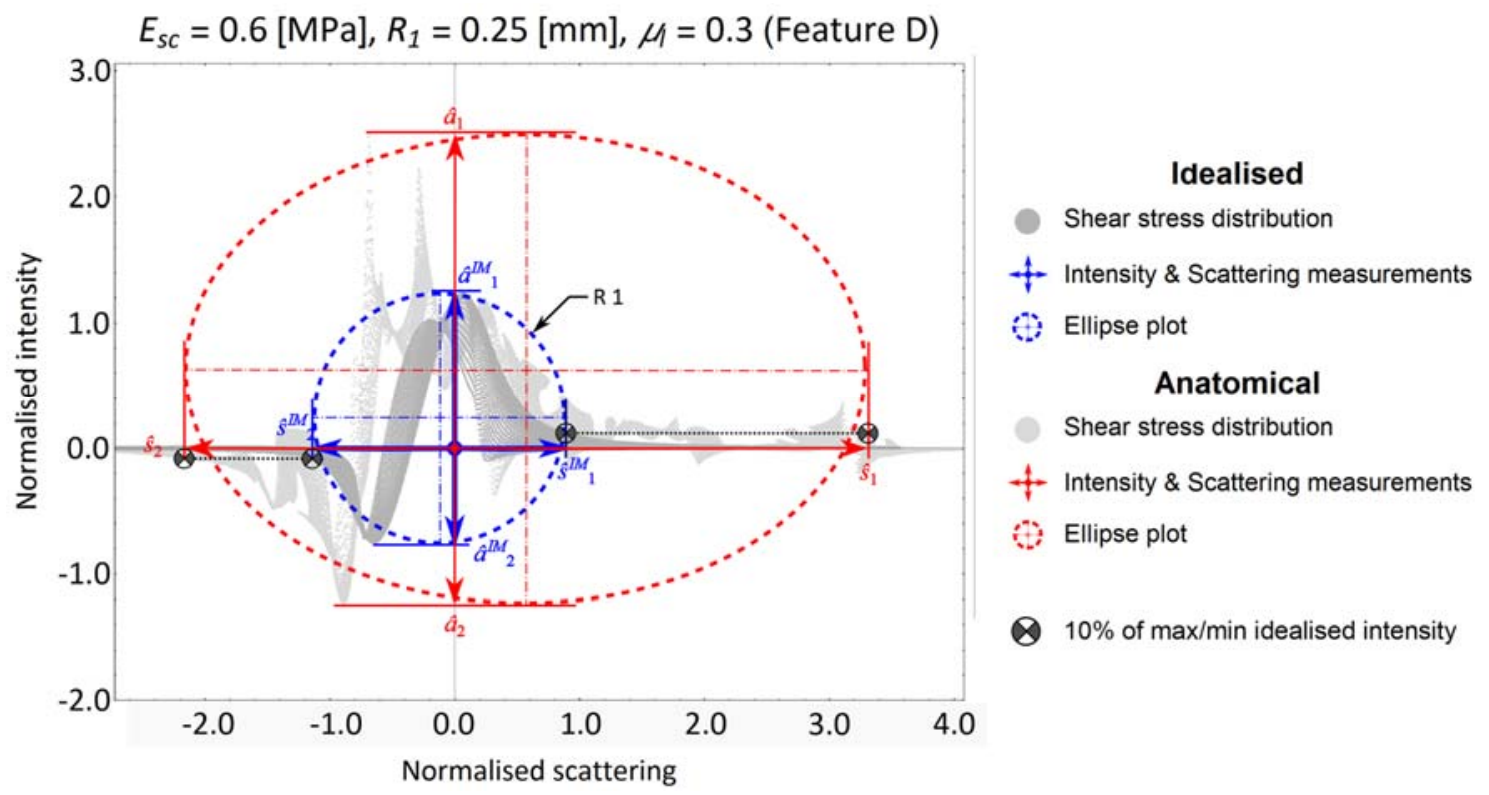

Fig. 4 Conceptual illustration of the ellipse plots described in Section $\mathbf{2 . 4}$ and used in Fig. $\mathbf{1 1}$ and Fig. $\mathbf{1 2}$ to visualise differences in shear stress intensity and scattering in the viable epidermis between the idealised (blue) and anatomical (red) models of the skin at any contacting location. Because these two metrics are given in normalised form with respect to the anatomical model, its results are represented by a circle of radius equal to 1 . 


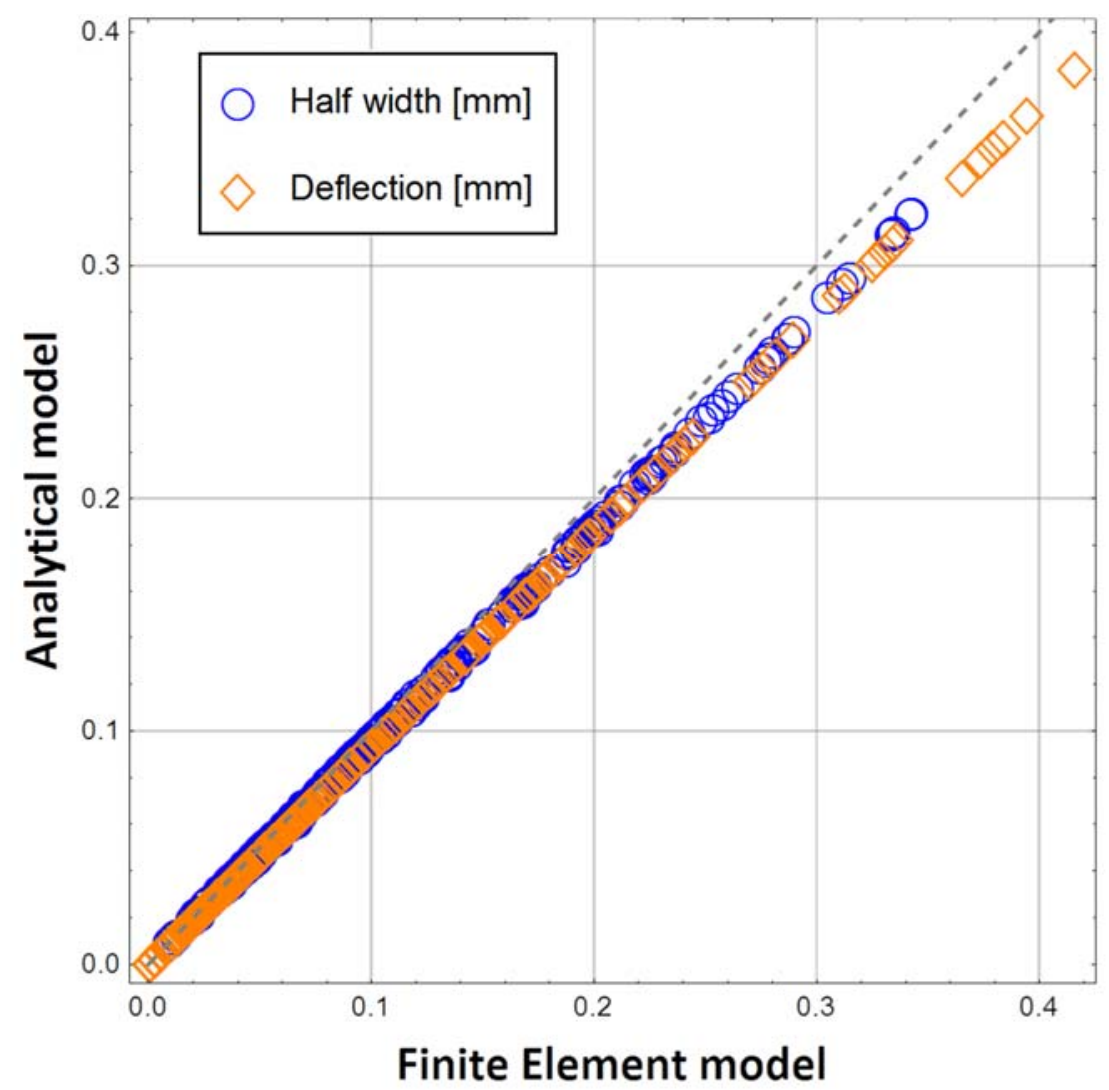

Fig. 5 Cross-validation plot of the analytical and finite element models of the idealised anatomical model for the half-width of the contact area and maximum deflection of the skin surface. 


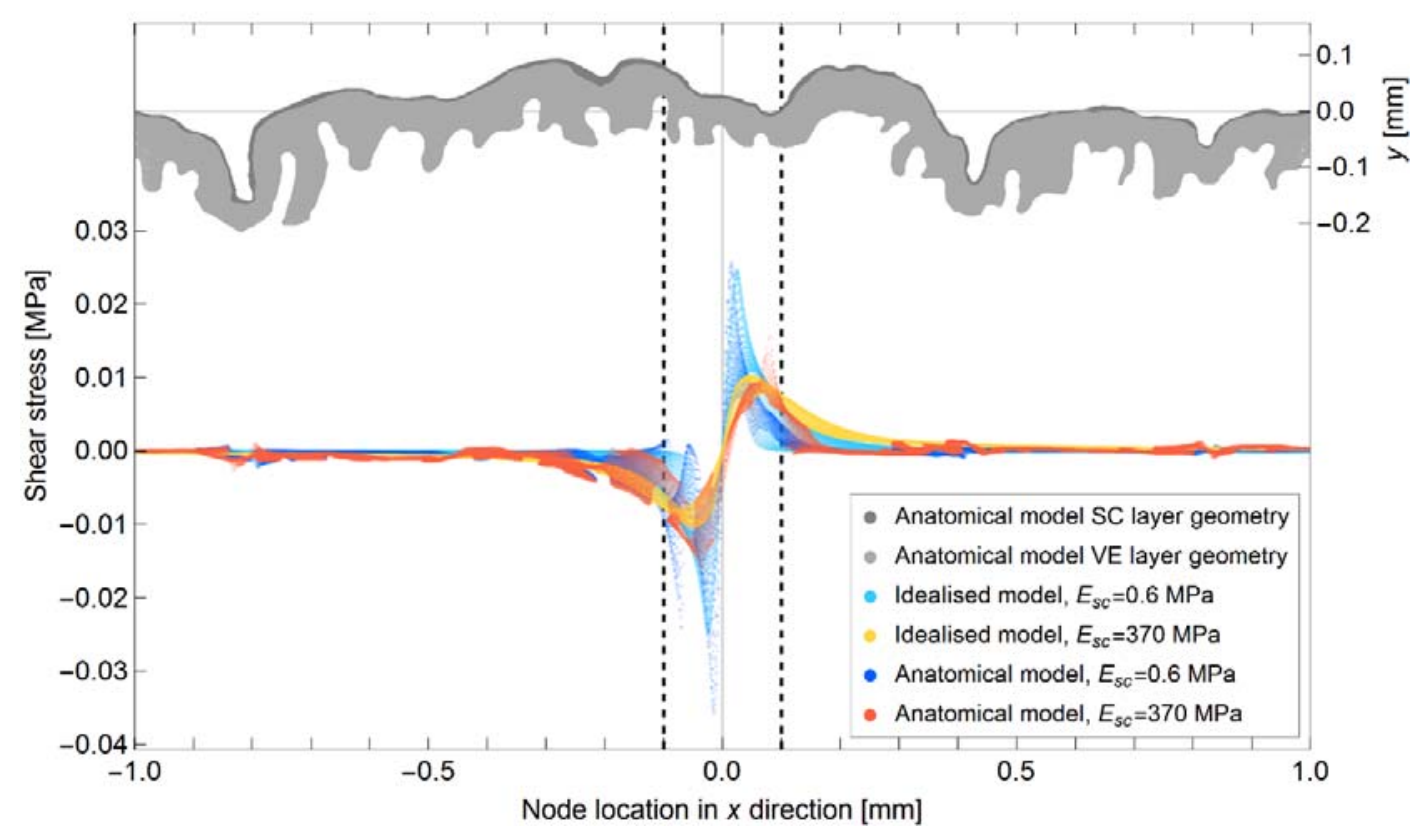

Fig. 6 Normal indentation case (frictionless) [ $R_{1}=0.1 \mathrm{~mm}, \mu_{l}=0.0$ and skin deflection is $\delta=23 \mu \mathrm{m}$ ]. Shear stress distribution in the viable epidermis contained within the region of interest (grey). The plot compares the shear stress distribution of the anatomical model (dark blue and orange) and that of the idealised model (light blue and yellow) as a function of the lateral position within the region of interest. The distance between the dotted lines represents the diameter of the indenter. 


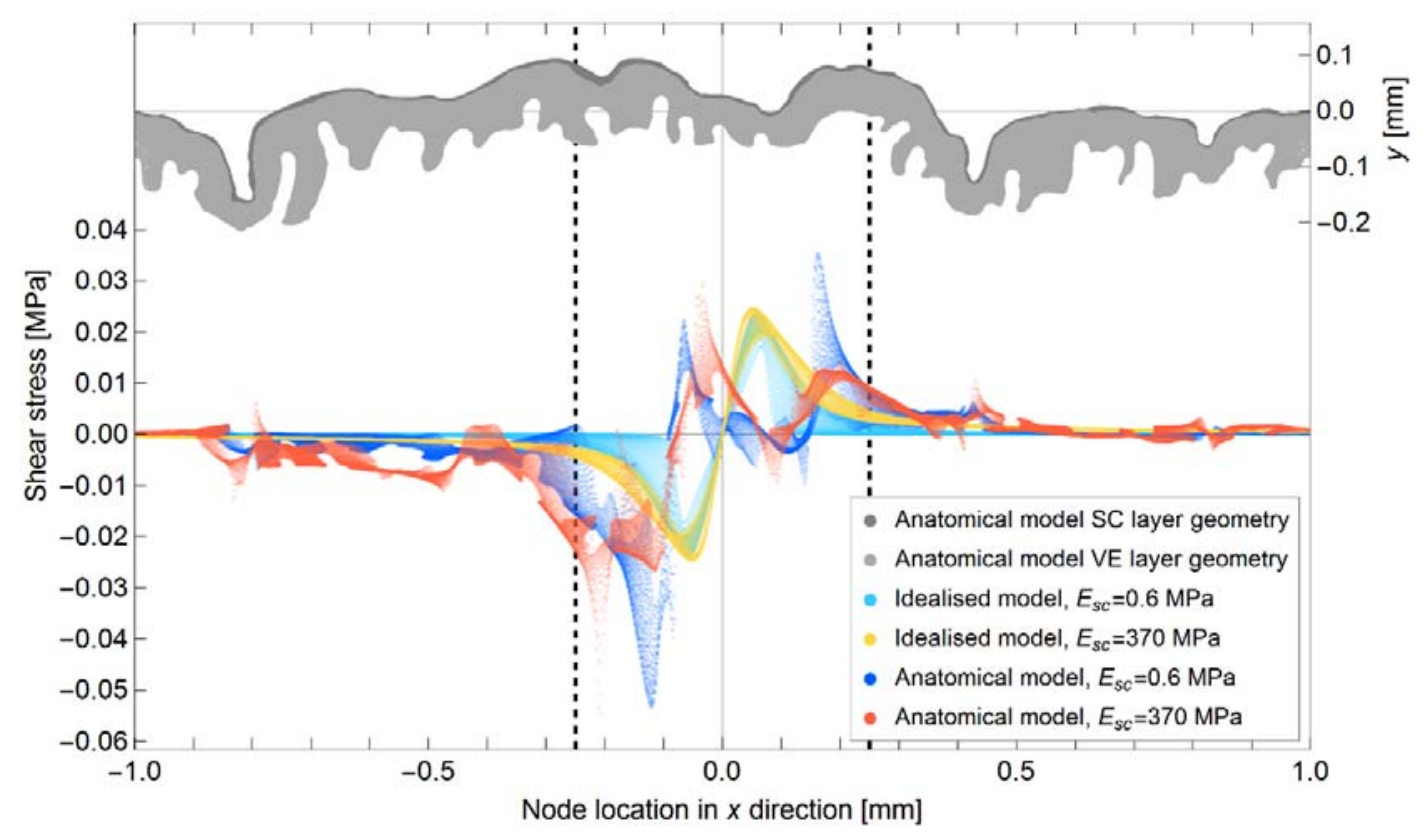

Fig. 7 Normal indentation case (frictionless) [ $R_{1}=0.25 \mathrm{~mm}, \mu_{l}=0.0$ and skin deflection is $\delta=50 \mu \mathrm{m}$ ]. Shear stress distribution in the viable epidermis contained within the region of interest (grey). The plot compares the shear stress distribution of the anatomical model (dark blue and orange) and that of the idealised model (light blue and yellow) as a function of the lateral position within the region of interest. The distance between the dotted lines represents the diameter of the indenter. 


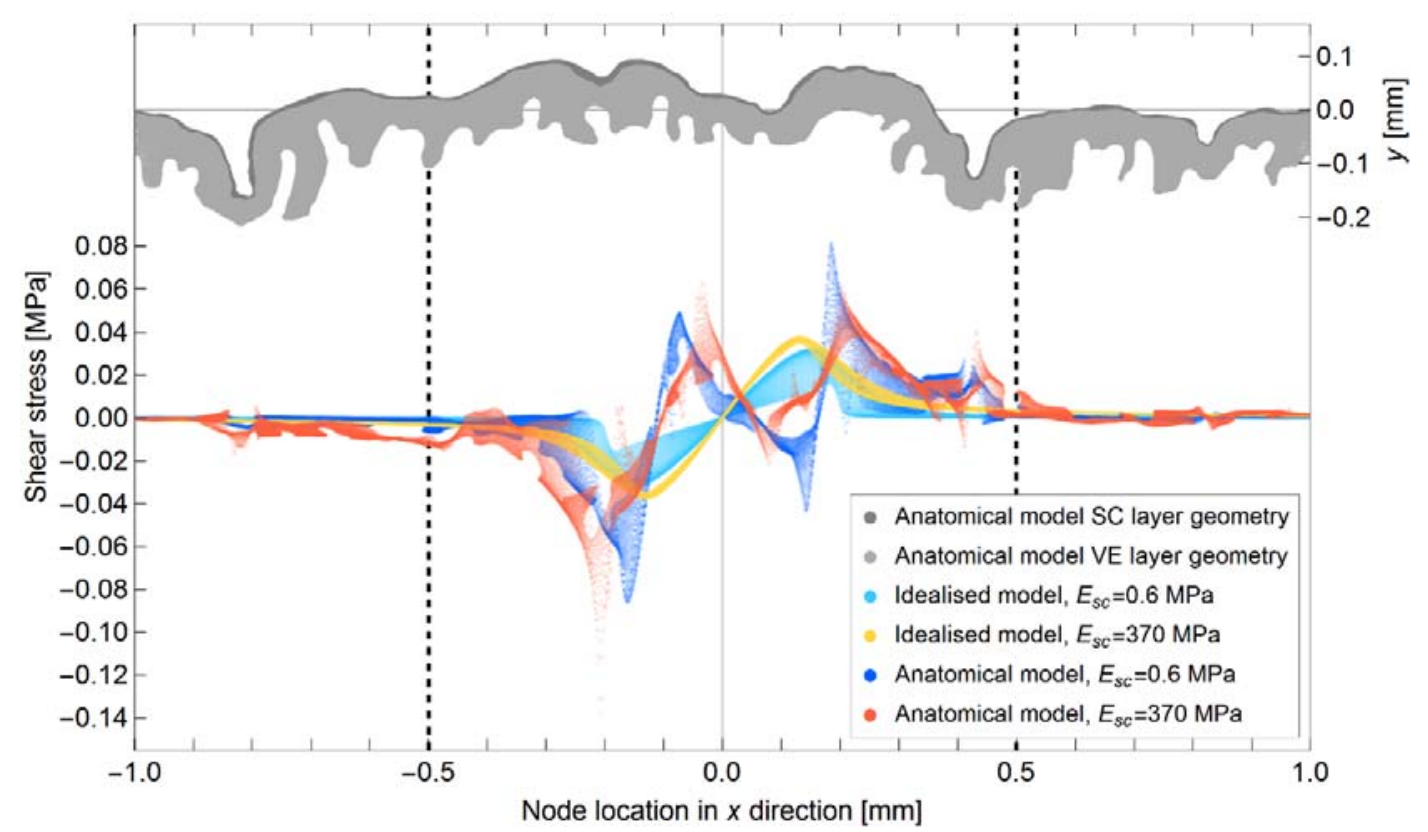

Fig. 8 Normal indentation case (frictionless) [ $R_{1}=0.5 \mathrm{~mm}, \mu_{l}=0.0$ and skin deflection is $\delta=120 \mu \mathrm{m}$ ]. Shear stress distribution in the viable epidermis contained within the region of interest (grey). The plot compares the shear stress distribution of the anatomical model (dark blue and orange) and that of the idealised model (light blue and yellow) as a function of the lateral position within the region of interest. The distance between the dotted lines represents the diameter of the indenter. 

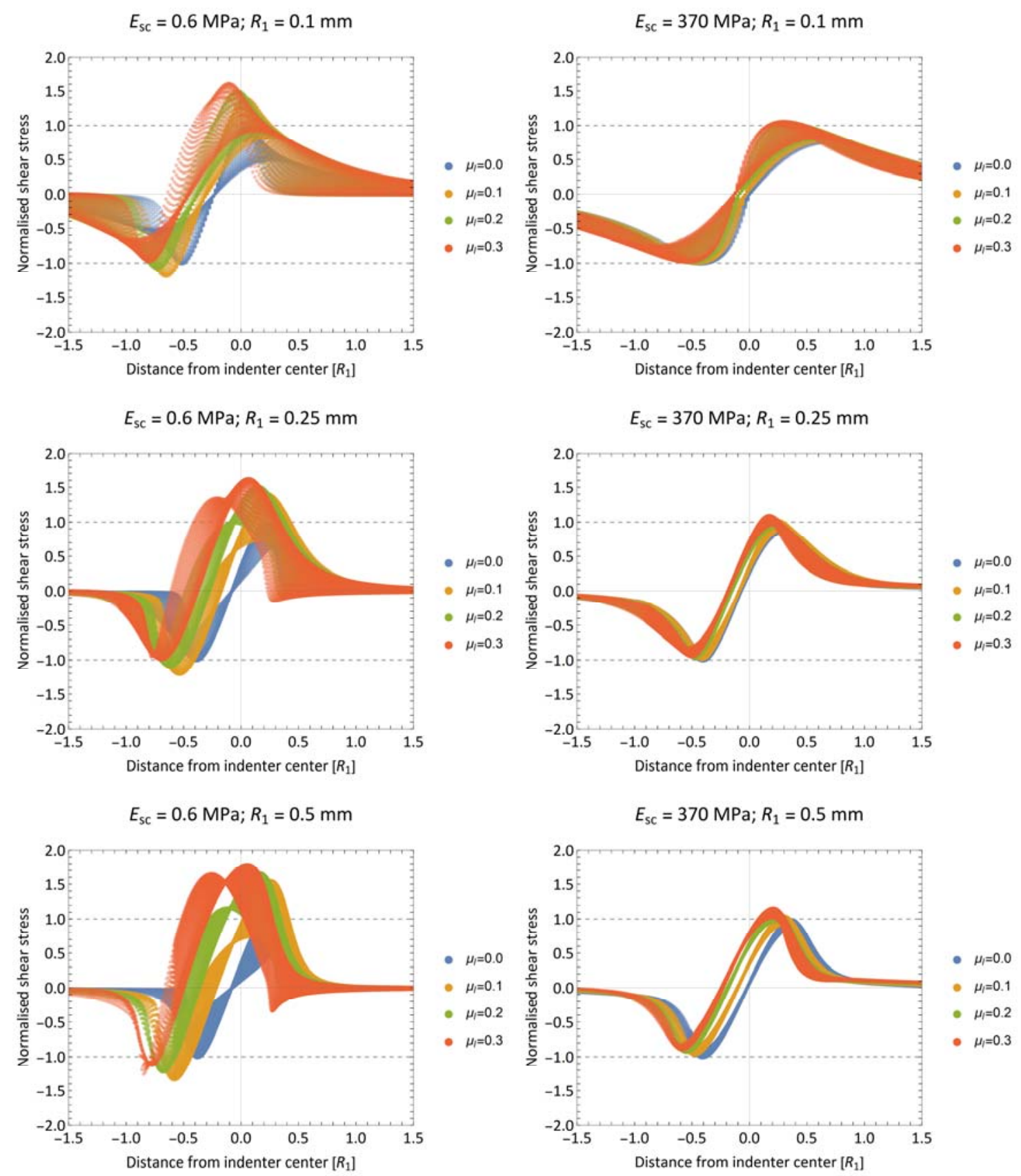

Fig. 9 Sliding indentation of the idealised model. Evolution of normalised shear stress distribution in the viable epidermis as function of the local coefficient of friction and lateral distance from the indenter centre. Computed shear stresses are normalised with respect to those obtained for the frictionless case (blue plot). Each plot corresponds to a particular combination of $R_{1}=\{0.1,0.25,0.5\}$ and $E_{S C}=\{0.6,370\}$. 


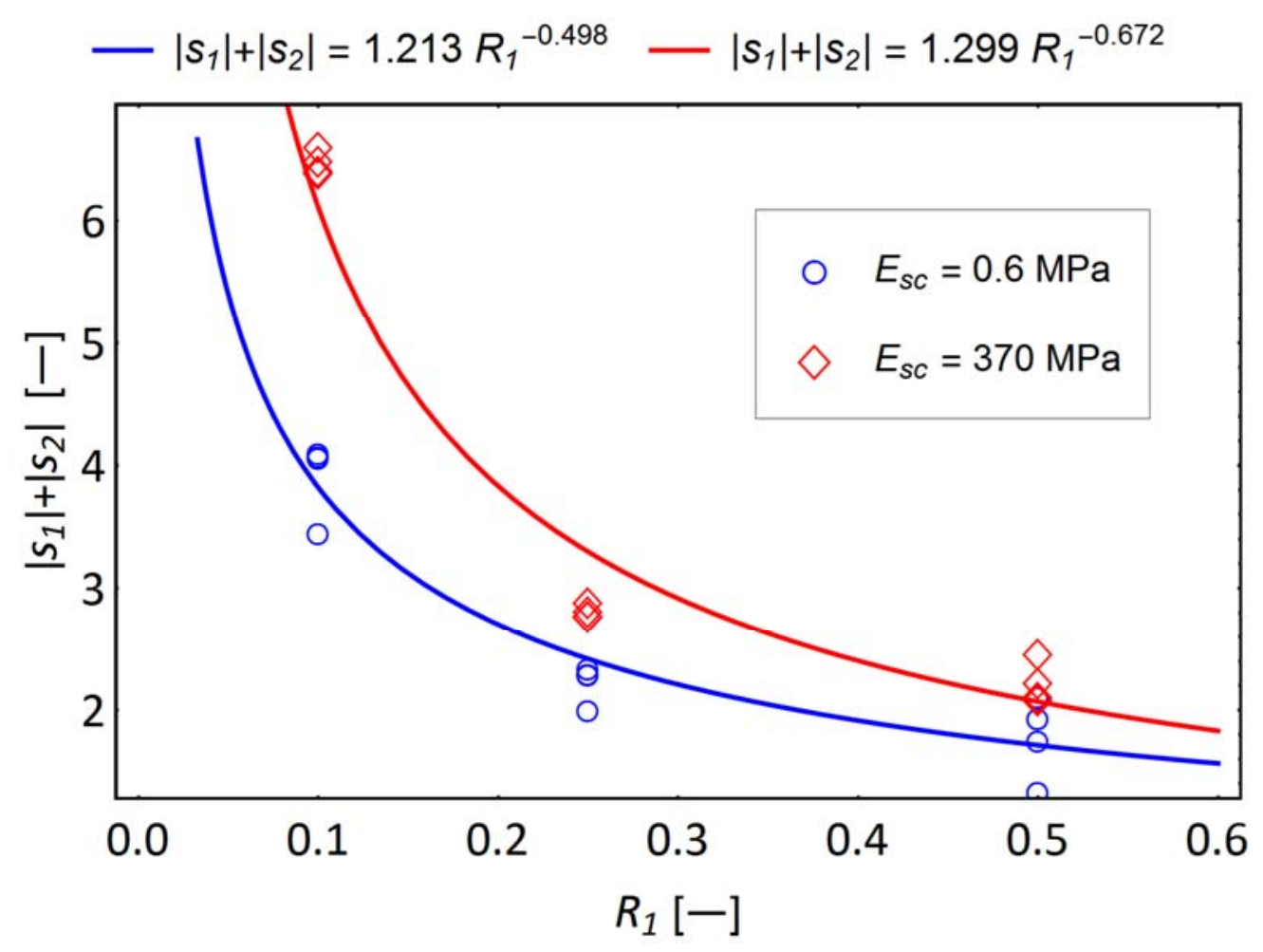

Fig. 10 Nonlinear fit of the shear stress propagation region $\left(\left|s_{1}\right|+\left|s_{2}\right|\right)$ to the indenter radius ( $\left.R_{1}\right)$ for the idealised sliding contact simulations with soft (blue) and stiff (red) stratum corneum. 


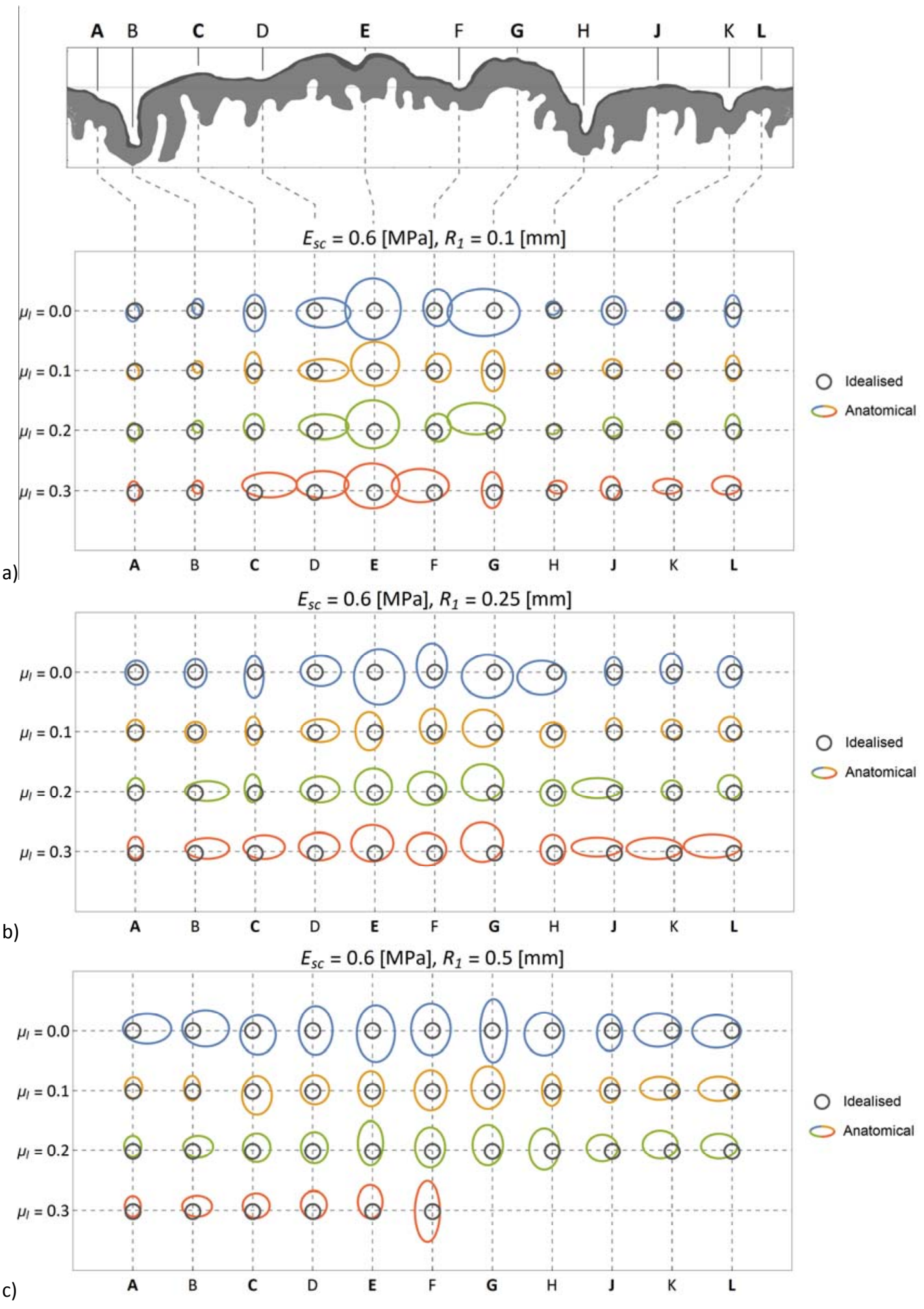

Fig. 11 Sliding indentation. Comparison of shear stress intensity and scattering at each anatomical feature (coloured ellipse) with respect to their corresponding idealised case (grey circle) for the simulations with indenter radii a) $R_{1}=0.10 \mathrm{~mm}, \mathrm{~b}$ ) $R_{1}=0.25 \mathrm{~mm}$ and c) $R_{1}=0.50 \mathrm{~mm}$, with stratum corneum Young's modulus $E_{S C}=0.6 \mathrm{MPa}$. For the case [ $E_{S C}=0.6$ $\mathrm{MPa}, R_{1}=0.5 \mathrm{~mm}, \mu_{l}=0.3$ ], the anatomical model simulations could only converge before the indenter reached feature $\mathrm{G}$ of the skin surface so the results are limited to features [A-F] (Fig. 12c). 


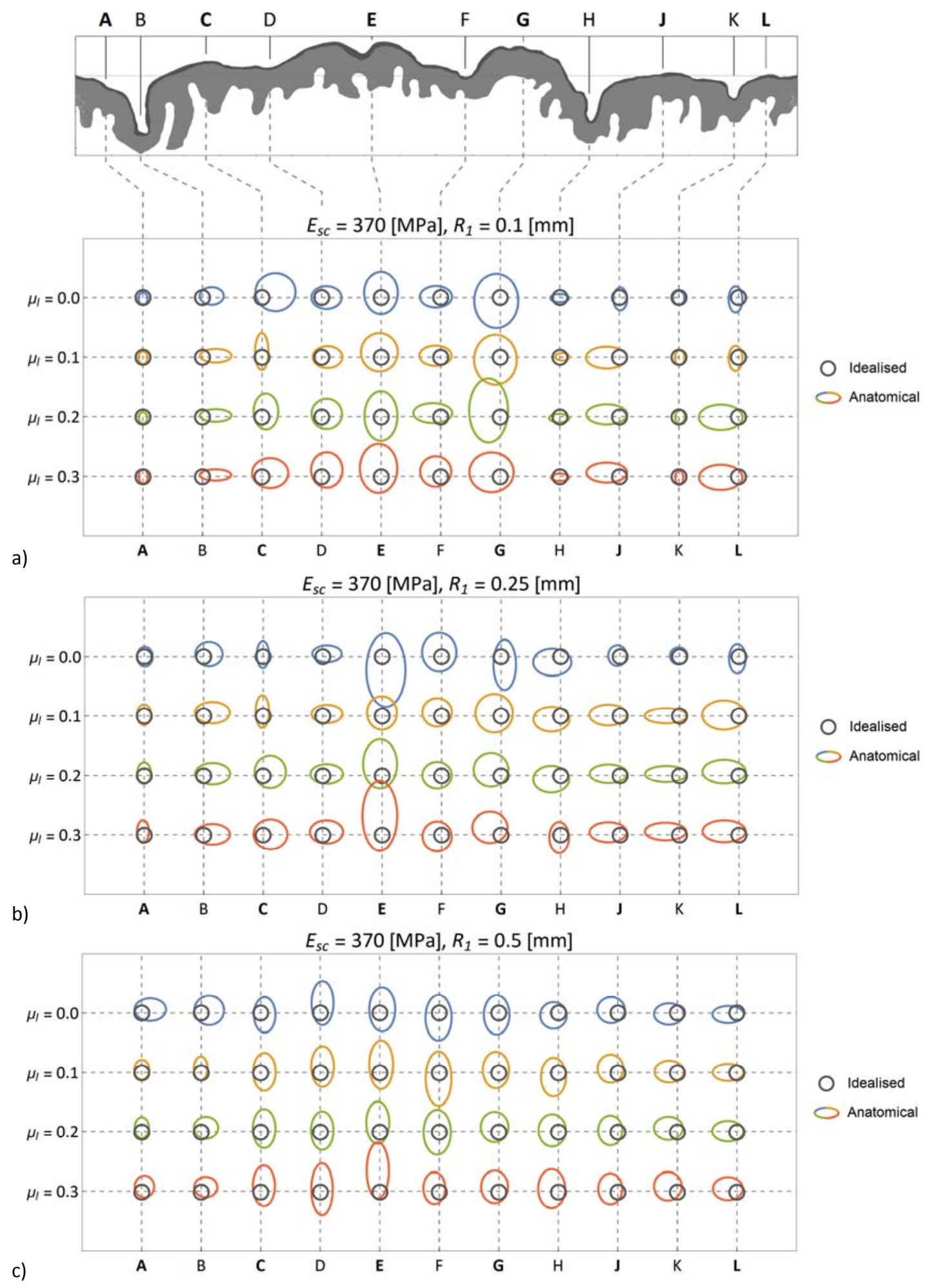

Fig. 12 Sliding indentation. Comparison of shear stress intensity and scattering at each anatomical feature (coloured ellipse) with respect to their corresponding idealised case (grey circle) for the simulations with indenter radii a) $R_{1}=0.10 \mathrm{~mm}, \mathrm{~b}$ ) $R_{1}=0.25 \mathrm{~mm}$ and c) $R_{1}=0.50 \mathrm{~mm}$, with stratum corneum Young's modulus $E_{S C}=370 \mathrm{MPa}$. 